\title{
Appendix: Synthesising evidence to estimate pandemic (2009) A/H1N1 influenza severity in 2009-2011
}

August 6, 2014

\section{Surveillance Data}

The influenza surveillance data available in the United Kingdom during each wave of the 2009 A/H1N1 pandemic are described below. The surveillance systems comprise a mixture of those already in place to monitor seasonal influenza prior to the pandemic, those set up during the first two waves in response to the pandemic, and those adapted or set up following the first two waves to monitor both seasonal and pandemic influenza.

\section{$1.1 \quad$ First two waves}

\subsubsection{Confirmed cases - estimating the time to hospitalisation}

The FF100 (Health Protection Agency et al., 2009) and FluZone (Health Protection Agency, 2010) databases of the first 7,088 confirmed cases provide data (Table 1) from which we can estimate the distribution of time to hospitalisation. The data extracted from the FF100/FluZone databases correspond to those confirmed cases with onset dates sampled between the 16th April 2009 and 2nd July 2009 (inclusive) and followed-up until the 13th July 2009 when they were administratively censored.

\begin{tabular}{lrrr}
\hline Age & Number of cases Number of hospitalisations & Proportion (\%) \\
\hline$<1$ & 113 & 11 & $9.73 \%$ \\
$1-4$ & 353 & 4 & $1.13 \%$ \\
$5-14$ & 3,054 & 20 & $0.65 \%$ \\
$15-24$ & 1,754 & 21 & $1.20 \%$ \\
$25-44$ & 1,339 & 20 & $1.49 \%$ \\
$45-64$ & 428 & 5 & $1.17 \%$ \\
$65+$ & 47 & 4 & $8.51 \%$ \\
\hline Total & 7,088 & 85 & $1.20 \%$ \\
\hline
\end{tabular}

Table 1: FF100/FluZone data on proportion of confirmed cases hospitalised.

A parametric mixture survival or cure rate model (Farewell, 1982) is used to estimate the distribution of time to hospitalisation over the follow-up period. In this model, we assume that the population of confirmed cases can be sub-divided into a sizable sub-population of cases who will never be hospitalised due to A/H1N1 influenza and a much smaller sub-population of symptomatic individuals at risk of experiencing the event of interest (i.e. hospitalisation). The proportion of confirmed cases that will result in hospitalisation corresponds to the confirmed case hospitalisation risk $(c C H R)$, also known as the mixing probability for belonging to the hospitalisation sub-population. Associated with this subpopulation is a time to hospitalisation which we model parametrically using a log-normal distribution. The gamma distribution for the time to hospitalisation was also considered and gave similar results for $c C H R$ and the mean time to hospitalisation. Information on time to recovery from onset for the sampled confirmed cases was unavailable. Since it was implausible to assume that those individuals yet to be admitted into hospital because of pandemic A/H1N1 were still potentially at risk of pandemic A/H1N1related admission after a significant time since symptom onset, we chose a threshold of 14 days after which 
individuals who had not yet been hospitalised were considered to have definitely recovered. Individuals who had not yet been hospitalised but whose observed "survival" times up to the 13th July 2009 were less than 14 days were considered to be potentially still at risk of hospitalisation (with probability equal to $c C H R$ ), with their observed times right censored. The analysis to estimate the distribution of time to hospitalisation was performed overall and stratified by age. The estimated case-hospitalisation risks overall and by age from the mixture models are shown in Table 2 ; they are nearly the same as the crude estimates in Table 1, but slightly higher because they take into account those cases with symptom onset on or after June 29 who might have been hospitalised if followed beyond July 13. Also shown are measures of location and spread for the time-to-hospitalisation distribution for those confirmed cases at risk of experiencing the event of interest (i.e. hospitalisation). These estimates are used as prior information in the severity model described in the main text and Section 2 below.

\begin{tabular}{lccccccr}
\hline Age & $c C H R$ & \multicolumn{2}{c}{$95 \%$ CI } & \multicolumn{4}{c}{ Time to event } \\
& & & & Mean & Median & SD & CV \\
\hline$<1$ & 0.0974 & 0.0517 & 0.1673 & 1.9861 & 1.6064 & 1.4439 & 0.7270 \\
$1-4$ & 0.0113 & 0.0043 & 0.0298 & 1.5574 & 0.9361 & 2.0702 & 1.3292 \\
$5-14$ & 0.0066 & 0.0043 & 0.0102 & 3.2159 & 1.6421 & 5.4150 & 1.6838 \\
$15-24$ & 0.0120 & 0.0079 & 0.0184 & 3.6167 & 2.3733 & 4.1584 & 1.1497 \\
$25-44$ & 0.0150 & 0.0097 & 0.0231 & 2.8781 & 1.6735 & 4.0268 & 1.3991 \\
$45-64$ & 0.0117 & 0.0049 & 0.0278 & 1.7117 & 1.2343 & 1.6446 & 0.9608 \\
$65+$ & 0.0851 & 0.0323 & 0.2058 & 1.2529 & 1.1360 & 0.5831 & 0.4654 \\
\hline Total & 0.0120 & 0.0097 & 0.0149 & 2.7725 & 1.6969 & 3.5826 & 1.2923 \\
\hline
\end{tabular}

Table 2: Estimated $c C H R$ and time to hospitalisation (mean, median, standard deviation, coefficient of variation) amongst those who are at risk of hospitalisation from the mixture model.

\subsubsection{Symptomatic cases}

The HPA stopped widespread laboratory confirmation of pandemic A/H1N1 cases at the end of June 2009, when a move was made to a containment phase. Only a proportion of cases continued to be laboratory-confirmed. During the pandemic, the HPA had access to data on the number of consultations for ILI in general practice (GP), stratified by age group $a$ and Strategic Health Authority (SHA) $r$ from the HPA/QSurveillance national system (Harcourt et al., 2011), covering a weekly population of approximately 23 million. During the first two waves of the pandemic, the HPA used weekly $(t)$ counts $y_{t, a, r}^{Q}$ of GP consultations for ILI and corresponding population denominators $n_{t, a, r}^{Q}$ from this source to estimate the number of symptomatic cases due to pandemic A/H1N1 (Health Protection Agency, 2009; Evans et al., 2011), as described briefly below.

The HPA also used data on GP consultations for ILI from the Royal College of General Practitioners (RCGP) Weekly Returns Service (Fleming, 1999), a sentinal network of GPs covering a weekly population of approximately 900,000. Respiratory (nose and throat) swabs are routinely taken from a sample of the patients observed in the RCGP scheme, as well as from those observed in the HPA Regional Microbiology Network (Health Protection Agency, 2010), covering a weekly population of approximately 400,000, and are virologically (i.e. by RT-PCR) tested for influenza strains and other respiratory infections. The week-, age- and region-specific virological positivity $p_{t, a, r}^{R}$, the proportion of patients consulting GPs for ILI who tested positive for the pandemic influenza strain, was estimated by the HPA using a logistic regression model (Health Protection Agency, 2009; Evans et al., 2011).

The week-, age- and region-specific number of symptomatic cases consulting GPs, $G_{t, a, r}$, was then estimated by multiplying the total size of the population of SHA $r$ aged $a, T_{a, r}$, by the GP consultation rate and by the estimate of virological positivity $\hat{p}_{t, a, r}^{R}$ :

$$
\hat{G}_{t, a, r}=T_{a, r} \hat{p}_{t, a, r}^{R} y_{t, a, r}^{Q} / n_{t, a, r}^{Q}
$$

The HPA also used data from the National Pandemic 'Flu Service (NPFS), a telephone and internet service set up to allow quick (self-)diagnosis, relieving burden on GPs (Health Protection Agency, 2010), on the number of anti-viral prescriptions made through the service, $n_{t, a, r}^{N}$. A subset of the patients 
prescribed anti-virals were tested for the pandemic influenza strain through self-swabbing, and these data were used to estimate the NPFS positivity $p_{t, a, r}^{N}$ through a further logistic regression (Health Protection Agency, 2009; Evans et al., 2011). The number of symptomatic cases prescribed anti-virals was then estimated as

$$
\hat{Y}_{t, a, r}=\hat{p}_{t, a, r}^{N} n_{t, a, r}^{N}
$$

Finally, the HPA estimated the total number of symptomatic pandemic cases as

$$
\hat{O}_{t, a, r, S}=\left(\hat{G}_{t, a, r}+\hat{Y}_{t, a, r}\right) / p_{G P}
$$

where $p_{G P}$ is the proportion of all symptomatic pandemic cases that consulted either a GP or the NPFS. As there were no direct data on this proportion available during the pandemic, $p_{G P}$ was assumed fixed and known at a central value of 0.5 . An "uncertainty range" was obtained by plugging in two extreme values $p_{G P}=0.3$ and $p_{G P}=0.7$ (Health Protection Agency, 2009; Evans et al., 2011). Denote the central estimate and the two extreme estimates by $\hat{O}_{t, a, r, S}$ and $O_{t, a, r, S}^{\text {low }}, O_{t, a, r, S}^{\text {high }}$ respectively (the sums $\hat{O}_{w, a, S}$ over weeks $t \in$ wave $w$ and SHAs in England and corresponding bounds $O_{w, a, S}^{l o w}$ and $O_{w, a, S}^{\text {high }}$ are given in Table 3). These estimates were used as "data" in the model of Presanis et al. (2011) to estimate severity in the first two waves, as described in the main text and Section 2 below.

\begin{tabular}{lrrrrrr}
\hline & & Wave 1 & & Wave 2 \\
Age $a$ & $\hat{O}_{w, a, S}$ & $O_{w, a, S}^{\text {low }}$ & $O_{w, a, S}^{\text {high }}$ & $\hat{O}_{w, a, S}$ & $O_{w, a, S}^{\text {low }}$ & $O_{w, a, S}^{\text {high }}$ \\
\hline$<1$ & 3,300 & 1,400 & 6,300 & 2,000 & 1,000 & 4,300 \\
$1-4$ & 17,700 & 7,600 & 34,500 & 25,000 & 12,500 & 54,200 \\
$5-14$ & 94,400 & 39,600 & 181,000 & 159,200 & 79,600 & 344,900 \\
$15-24$ & 76,500 & 33,600 & 151,400 & 92,700 & 46,400 & 201,000 \\
$25-44$ & 75,400 & 33,300 & 149,900 & 118,400 & 59,200 & 256,500 \\
$45-64$ & 20,000 & 8,800 & 39,600 & 51,700 & 25,800 & 112,000 \\
$65+$ & 1,900 & 800 & 3,800 & 3,000 & 1,500 & 6,400 \\
\hline Total & 289,300 & 125,100 & 566,400 & 452,000 & 226,000 & 979,300 \\
\hline
\end{tabular}

Table 3: Estimated numbers (rounded to the nearest 100) of symptomatic pandemic A/H1N1 cases from June to August 2009 (Wave 1) and September 2009 to February 2010 (Wave 2), England, reported by the HPA.

\subsubsection{Sero-prevalence data}

Residual serum samples from patients accessing health-care in England are collected annually by the HPA sero-epidemiology programme in order to carry out cross-sectional studies of antibody prevalence (Osborne et al., 2000). Data from a cross-sectional survey taken pre-pandemic (as a baseline in 2008), post-first-wave in August and September 2009 (Miller et al., 2010) and post-second-wave in February 2010 (Hardelid et al., 2011) are available (Table 4). They provide evidence on the age-specific proportion of the population who may have been infected (with or without symptoms) during the first and second waves of infection. This is based on assuming the proportion of study participants with haemagglutination inhibition titre greater or equal to $1: 32$ is a measure of pandemic A/H1N1 antibody prevalence. Note that infection is assumed to have occurred a fixed lag prior to the date a sample is taken.

Note that the raw data imply "negative" incidence rates in some age groups, perhaps due to small sample sizes or biases in the sampled population. Within the model, we constrain the proportion of the population infected (the attack rate) to be positive (see Section 2 below). Although residual sera are now available from pre- and post- the third wave (Hoschler et al., 2012), further work is still required to use these data while accounting for vaccination, to distinguish between individuals testing sero-positive due to infection and to vaccination. The third wave data are not therefore used in this paper.

\subsubsection{Hospitalisations - web-based surveillance system}

The data on laboratory-confirmed hospitalisations (Campbell et al., 2011) for pandemic A/H1N1 are stratified into the first two waves of infection by date of illness onset: the first wave comprises onset 


\begin{tabular}{lrrrrrrrrr}
\hline & & \multicolumn{2}{c}{ Baseline } & \multicolumn{3}{c}{ Post-first wave } & \multicolumn{3}{c}{ Post-second wave } \\
Age $a$ & Positive & Tested & $\%$ & Positive & Tested & $\%$ & Positive & Tested & $\%$ \\
\hline$<1$ & 1 & 46 & $2.17 \%$ & 8 & 78 & $10.26 \%$ & 21 & 159 & $13.21 \%$ \\
$1-4$ & 2 & 125 & $1.60 \%$ & 8 & 99 & $8.08 \%$ & 81 & 237 & $34.18 \%$ \\
$5-14$ & 7 & 188 & $3.72 \%$ & 38 & 266 & $14.29 \%$ & 329 & 722 & $45.57 \%$ \\
$15-24$ & 21 & 120 & $17.50 \%$ & 36 & 256 & $14.06 \%$ & 177 & 495 & $35.76 \%$ \\
$25-44$ & 14 & 158 & $8.86 \%$ & 50 & 407 & $12.29 \%$ & 207 & 787 & $26.30 \%$ \\
$45-64$ & 31 & 217 & $14.29 \%$ & 44 & 470 & $9.36 \%$ & 133 & 515 & $25.83 \%$ \\
$65+$ & 128 & 549 & $23.32 \%$ & 99 & 538 & $18.40 \%$ & 215 & 747 & $28.78 \%$ \\
\hline Total & 204 & 1,403 & $14.54 \%$ & 283 & 2,114 & $13.39 \%$ & 1,163 & 3,662 & $31.76 \%$ \\
\hline
\end{tabular}

Table 4: Number of serum samples tested and found positive for antibodies to pandemic A/H1N1.

dates from 26th May - 31st August 2009; the second comprises onset dates from 1st September 2009 28th February 2010. The number of hospitalisations by period $w$ and age $a$ is shown in Table 5, denoted $O_{w, a, H}$.

\begin{tabular}{|c|c|c|}
\hline Age & $\begin{array}{l}\text { Number of ho } \\
\text { Wave } 1 O_{1, a, H}\end{array}$ & $\begin{array}{l}\text { ospitalisations } \\
\text { Wave } 2 O_{2, a, H}\end{array}$ \\
\hline$<1$ & 79 & 100 \\
\hline $1-4$ & 115 & 175 \\
\hline $5-14$ & 201 & 259 \\
\hline $15-24$ & 183 & 211 \\
\hline $25-44$ & 224 & 300 \\
\hline $45-64$ & 132 & 205 \\
\hline $65+$ & 36 & 56 \\
\hline Total & 970 & 1,306 \\
\hline
\end{tabular}

Table 5: Number of hospitalisations of laboratory-confirmed pandemic A/H1N1 observed in web-based hospital surveillance system as at 9th July 2010, by period of symptom onset, England.

For the two subsets of hospitalisation data with non-missing data on ICU admission and final outcome (discharge, transfer or death) respectively, the observed proportions admitted to ICU and ending in death are given in Tables 6 and 7 respectively. Denote the subset of 1,411 (62\% of the total) hospitalisations in Table 6 (595 in wave 1, 816 in wave 2) by the superscript WS1 and the subset of 2,133 (94\% of the total) hospitalisations in Table 7 (864 in wave 1, 1,269 in wave 2) by the superscript WS2.

\begin{tabular}{lrrrrrr}
\hline & & Wave 1 & & \multicolumn{2}{c}{ Wave 2} \\
Age $a$ & ICU admissions & Hospitalisations & $\%$ & ICU admissions & Hospitalisations & $\%$ \\
& $O_{1, a, I}^{W S S 1}$ & $O_{1, a, H}^{W S 1}$ & & $O_{2, a, I}^{W S 1}$ & $O_{2, a, H}^{W S S 1}$ & \\
\hline$<1$ & 0 & 41 & $0.0 \%$ & 4 & 68 & $5.9 \%$ \\
$1-4$ & 0 & 72 & $0.0 \%$ & 2 & 117 & $1.7 \%$ \\
$5-14$ & 7 & 109 & $6.4 \%$ & 12 & 179 & $6.7 \%$ \\
$15-24$ & 15 & 125 & $12.0 \%$ & 15 & 126 & $11.9 \%$ \\
$25-44$ & 16 & 139 & $11.5 \%$ & 43 & 173 & $24.9 \%$ \\
$45-64$ & 16 & 91 & $17.6 \%$ & 44 & 116 & $37.9 \%$ \\
$65+$ & 0 & 18 & $0.0 \%$ & 10 & 37 & $27.0 \%$ \\
\hline Total & 54 & 595 & $9.1 \%$ & 130 & 816 & $15.9 \%$ \\
\hline
\end{tabular}

Table 6: Proportion of hospitalisations resulting in ICU admission observed in web-based hospital surveillance system of laboratory-confirmed pandemic A/H1N1 cases as at 9th July 2010, by period of symptom onset, England. 


\begin{tabular}{lrrrrrr}
\hline Age $a$ & \multicolumn{3}{c}{ Wave 1} & \multicolumn{3}{c}{ Wave 2} \\
& $\begin{array}{r}\text { Deaths } \\
O_{1, a, D}^{W S 2}\end{array}$ & $\begin{array}{r}\text { Hospitalisations } \\
O_{1, a, H}^{W S 1}\end{array}$ & $\%$ & $\begin{array}{r}\text { Deaths } \\
O_{2, a, D}^{W S 2}\end{array}$ & $\begin{array}{r}\text { Hospitalisations } \\
O_{2, a, H}^{W S 1}\end{array}$ & $\%$ \\
\hline$<1$ & 1 & 71 & $1.4 \%$ & 0 & 99 & $0.0 \%$ \\
$1-4$ & 0 & 107 & $0.0 \%$ & 1 & 174 & $0.6 \%$ \\
$5-14$ & 3 & 178 & $1.7 \%$ & 3 & 256 & $1.2 \%$ \\
$15-24$ & 2 & 164 & $1.2 \%$ & 6 & 206 & $2.9 \%$ \\
$25-44$ & 4 & 197 & $2.0 \%$ & 13 & 288 & $4.5 \%$ \\
$45-64$ & 6 & 116 & $5.2 \%$ & 17 & 195 & $8.7 \%$ \\
$65+$ & 3 & 31 & $9.7 \%$ & 10 & 51 & $19.6 \%$ \\
\hline Total & 19 & 864 & $2.2 \%$ & 50 & 1,269 & $3.9 \%$ \\
\hline
\end{tabular}

Table 7: Proportion of hospitalisations resulting in death observed in web-based hospital surveillance system of laboratory-confirmed pandemic A/H1N1 cases as at 9th July 2010, by period of symptom onset, England.

\subsubsection{Deaths}

As of 9th July 2010, 381 deaths amongst persons in England with confirmed pandemic A/H1N1 had been reported (Donaldson et al., 2009; Pebody et al., 2010) to the HPA and/or the Chief Medical Officer (CMO). Table 8 gives the number of observed deaths, denoted $O_{w, a, D}$, by wave $w$ and age group $a$ : deaths among individuals with symptom onset in 1st June - 31st August 2009 are defined as deaths due to the first wave; deaths among individuals with onset date in 1st September 2009 - 28th February 2010 are defined as deaths due to the second wave. Note that although the 381 observed deaths occur among individuals with confirmed infection, the deaths may not necessarily be attributable to pandemic A/H1N1. It is assumed that all deaths occurred from within hospitals. The deaths observed in the web-based surveillance system (Table 7) are assumed a subset of the total deaths occurring in hospital (Table 8).

\begin{tabular}{|c|c|c|c|}
\hline Age & \multicolumn{3}{|c|}{ Number of deaths } \\
\hline$<1$ & 2 & 6 & 8 \\
\hline $1-4$ & 2 & 13 & 11 \\
\hline $5-14$ & 10 & 28 & 14 \\
\hline $15-24$ & 8 & 28 & 18 \\
\hline $25-44$ & 16 & 78 & 106 \\
\hline $45-64$ & 23 & 103 & 197 \\
\hline $65+$ & 18 & 45 & 81 \\
\hline Total & 79 & 301 & 435 \\
\hline
\end{tabular}

Table 8: Deaths in cases with laboratory-confirmed pandemic A/H1N1 reported to the HPA and/or the CMO, as at 9th July 2010 (for the first two waves) and at 4th May 2011 (third wave), England.

During the third wave, 435 deaths in cases with laboratory confirmed pandemic A/H1N1 infection had been reported to the HPA and/or the CMO by 4th May 2011.

\subsection{Third wave data}

\subsubsection{Primary care consultations and positivity}

As data on GP consultations from the same surveillance source were not available at the same level of disaggregation during the third wave, instead, corresponding data from the RCGP Weekly Returns Service (Fleming, 1999) were used to inform GP consultation rates for ILI. The observed weekly $(t)$ and age-specific $(a)$ number of consultations for an ILI, $y_{t, a}^{G P}$, and corresponding population denominators, 
$n_{t, a}^{G P}$, covered by the practices in the scheme, are summarised by the weekly ILI consultation rates per 100,000 population shown in Figure 1.

RCGP consultation data

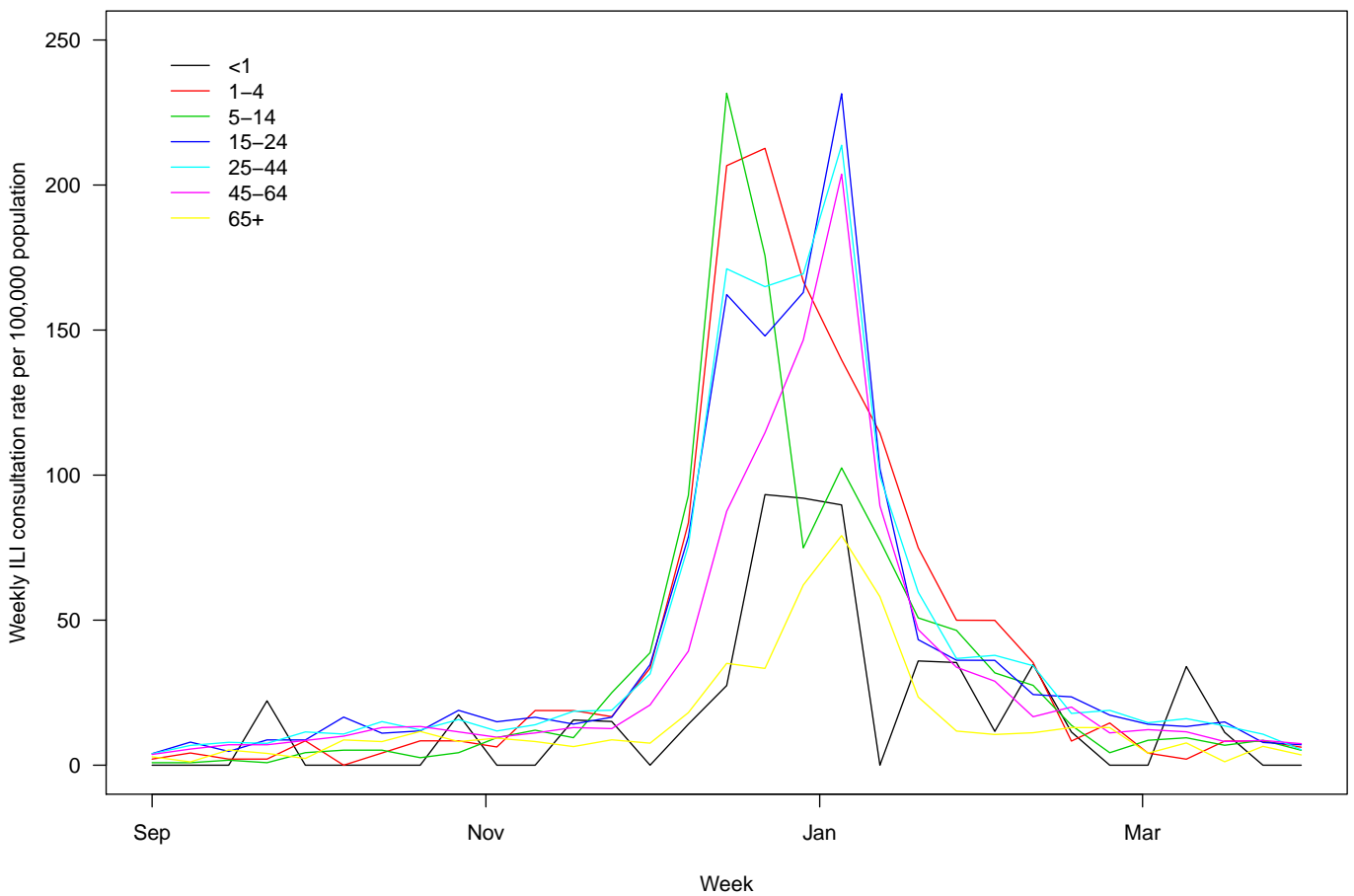

Figure 1: Observed weekly ILI consultation rate per 100,000 population from the RCGP Weekly Returns Service, by age group, in the period September 2010 to March 2011.

Two alternative sets of virological positivity data (tests positive for pandemic A/H1N1 out of number of tests performed, $\left.\left(y_{t, a}^{R}, n_{t, a}^{R}\right)\right)$, were considered. In the main analysis, test results from respiratory swabs taken in both the RCGP (Fleming, 1999) and the RMN (Health Protection Agency, 2010) schemes (as in the first two waves, Section 1.1.2) were used. In a sensitivity analysis, only swabs for which we have information on both date of swab and date of ILI consultation, and which were taken within 5 days of the consultation, were used. The observed positivity from the two sets of data are shown in Figure 2. Note that in general, the swabs taken within 5 days of a consultation tend to show higher positivity, but are available for a shorter period of time: the positivity observed in some age groups in the full RCGP/RMN data in the early period (September/October) are not observed in the restricted dataset.

As in the first two waves, datasets on GP consultations for ILI and virological positivity may be combined to estimate the cumulative number of symptomatic infections that occurred in the third wave. However, in contrast to the HPA method of section 1.1.2, here a fully Bayesian regression on time and age (c.f. Birrell et al., 2011) is fitted jointly to the data $\left(y_{t, a}^{G P}, n_{t, a}^{G P}, y_{t, a}^{R}, n_{t, a}^{R}\right)$, to estimate the weekly rate of ILI consultation that is due to the pandemic strain of influenza. This model and its results are described below in Section 3.1, with the results of the main analysis using the full RCGP/RMN positivity data in Table 13, and the results of the alternative sensitivity analysis using the restricted data in Table 14 .

\subsubsection{Hospitalisation data}

During the 2010/11 influenza season (i.e. during which the third wave of pandemic A/H1N1 infection was experienced), the web-based system of Section 1.1.4 evolved into a pilot sentinel scheme for hospital surveillance, the UK Severe Influenza Surveillance System (USISS, http://www.hpa.org.uk/ Publications/InfectiousDiseases/Influenza/1105influenzareport/). In England, 23 acute NHS trusts were recruited to report weekly aggregate numbers of confirmed cases hospitalised and admitted to ICU. The sums over weeks in the third wave of these numbers $\left(O_{3, b, H}\right.$ and $O_{3, b, I}$ respectively), by age 

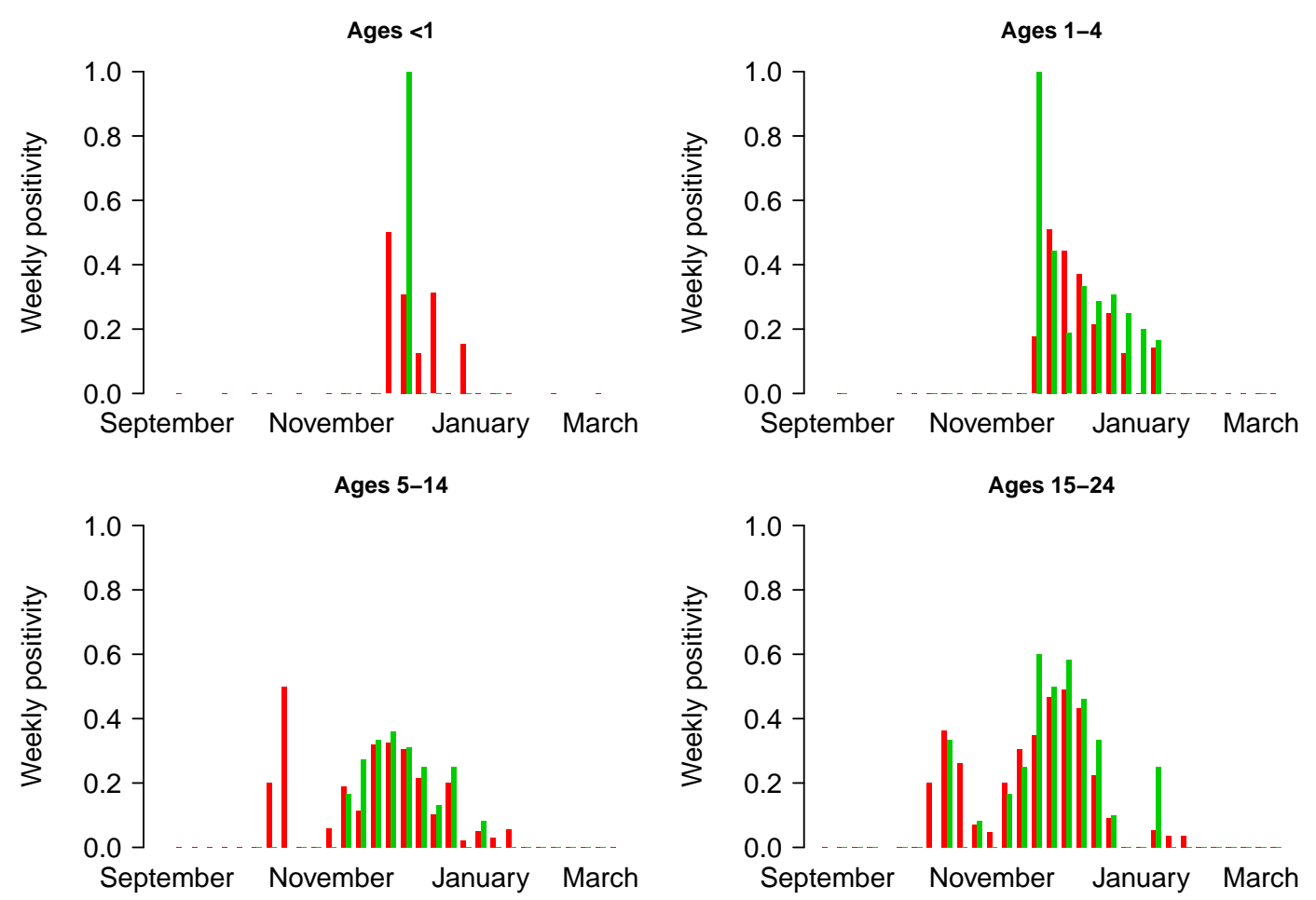

Ages 25-44

Ages 45-64
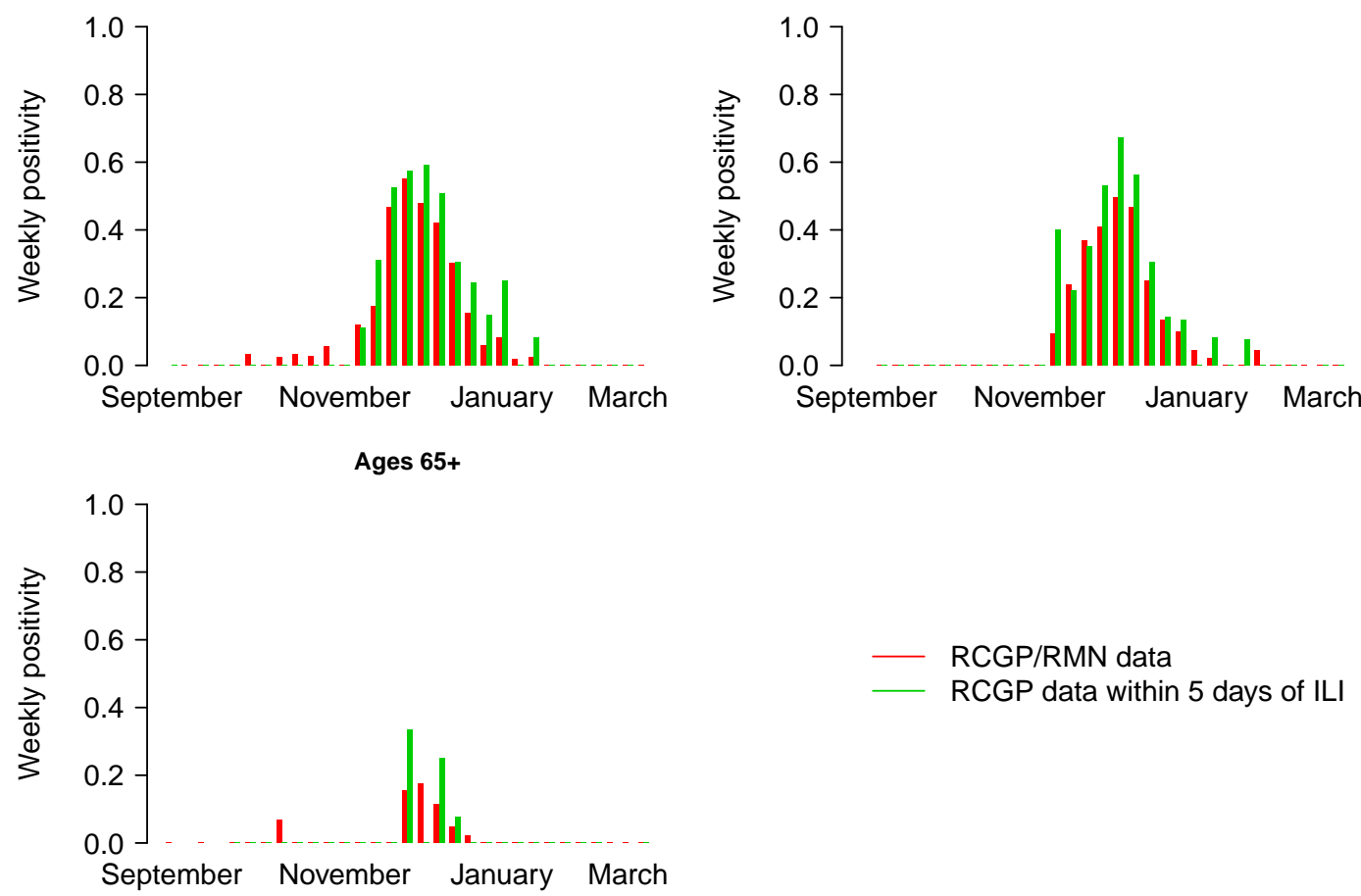

Figure 2: Observed positivity for pandemic A/H1N1, by age group and surveillance scheme (RCGP/RMN or RCGP restricted to swabs taken within 5 days of an ILI consultation), in the period September 2010 to March 2011. 
group $b \in\{<5,5-14,15-44,45-64,65+\}$, are given in Table 9 . Note we use the index $b$ to differentiate the age groups for which these data are available from the age groups in the severity model.

\begin{tabular}{rrrr}
\hline Age group & Hospitalisations & ICU admissions & $\%$ \\
$b$ & $O_{3, b, H}$ & $O_{3, b, I}$ & $O_{3, b, I} / O_{3, b, H}$ \\
\hline$<5$ & 215 & 9 & $4.19 \%$ \\
$5-14$ & 40 & 3 & $7.50 \%$ \\
$15-44$ & 571 & 103 & $18.04 \%$ \\
$45-64$ & 326 & 81 & $24.85 \%$ \\
$65+$ & 110 & 10 & $9.09 \%$ \\
\hline Total & 1,262 & 206 & 16.32 \\
\hline
\end{tabular}

Table 9: Confirmed cases of pandemic A/H1N1 in hospital and ICU reported to the USISS sentinel surveillance system (third wave), England.

\subsubsection{Prevalence-type ICU data}

Additionally to the USISS system, weekly numbers of ICU beds occupied by patients with suspected as opposed to confirmed pandemic A/H1N1 infection were available during the third wave from the Department of Health's Winter Watch scheme (Department of Health, 2011) covering all ICUs in England during the 2010-11 season. We have observations, denoted $y_{t, b, I}$, of the number of suspected cases present in ICU, by age group $b$ and week $t$. Throughout the following, we consider either one of two age groups, children (age $b \leq 16$ ) or adults (age $b>16$ ). Observations are available once a week, at the starts of weeks 50 to 8 in the 2010/11 season, i.e. from 15th Dec 2010 to 23rd Feb 2011). These data are given in Table 10.

\begin{tabular}{rrr}
\hline Week & Children & Adults \\
$t$ & $y_{t, \text { child,I }}$ & $y_{t, \text { adult }, I}$ \\
\hline 50 & 19 & 163 \\
51 & 43 & 417 \\
52 & 66 & 672 \\
1 & 47 & 736 \\
2 & 26 & 635 \\
3 & 26 & 392 \\
4 & 13 & 234 \\
5 & 10 & 150 \\
6 & 6 & 93 \\
7 & 2 & 69 \\
8 & 3 & 55 \\
\hline
\end{tabular}

Table 10: Observed in ICU with suspected pandemic A/H1N1 influenza. Source: DH website http://winterwatch.dh.gov.uk/

These ICU data on suspected cases may be modelled jointly with an appropriate source of virological positivity data to estimate the cumulative number of ICU admissions due to pandemic A/H1N1 influenza (Section 4.2). DataMart is such a positivity data source: it comprises a sentinel laboratory surveillance system monitoring virological testing for influenza and other respiratory viruses in 16 HPA and NHS laboratories in England (http://www . hpa .org.uk/Publications/InfectiousDiseases/ Influenza/1105influenzareport/). The laboratories test swabs from both primary and secondary care for a variety of respiratory viruses and provide data on both the number of tests and the number of tests positive for different strains of these viruses, including pandemic A/H1N1 during the third wave. Since the system collects data from secondary care as well as primary care, the data are thought to be representative of more severe cases of pandemic A/H1N1 than the RCGP system (Section 1.2.1). However, since the system does include primary care, we take the observed pandemic A/H1N1 positivity to provide 
a lower bound for the proportion of hospitalised suspected cases that are true (confirmed) cases (Section 4.2). The weekly number of tests $\left(n_{t, b}^{D M}\right)$ and tests positive $\left(y \cdot y_{t, b}^{D M}\right)$ by age group $b \in\{<15,15+\}$ are given in Table 11.

\begin{tabular}{|c|c|c|c|c|c|c|}
\hline Week & Chi & dren & $<15$ & Adv & ilts & $=15$ \\
\hline & 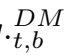 & & $y / n$ & $y_{t, b}^{D M}$ & $n_{t, b}^{D M}$ & $y / n$ \\
\hline 50 & 312 & 1,168 & $26.71 \%$ & 1,072 & 2,682 & $39.97 \%$ \\
\hline 51 & 500 & 1,611 & $31.04 \%$ & 1,788 & 4,401 & $40.63 \%$ \\
\hline 52 & 420 & 1,468 & $28.61 \%$ & 1,843 & 4,914 & $37.51 \%$ \\
\hline 1 & 247 & 1,204 & $20.51 \%$ & 926 & 3,962 & $23.37 \%$ \\
\hline 2 & 139 & 1,018 & $13.65 \%$ & 391 & 3,012 & $12.98 \%$ \\
\hline 3 & 56 & 882 & $6.35 \%$ & 132 & 1,749 & $7.55 \%$ \\
\hline 4 & 21 & 805 & $2.61 \%$ & 63 & 1,239 & $5.08 \%$ \\
\hline 5 & 9 & 685 & $1.31 \%$ & 33 & 858 & $3.85 \%$ \\
\hline 6 & 4 & 518 & $0.77 \%$ & 27 & 703 & $3.84 \%$ \\
\hline 7 & 3 & 405 & $0.74 \%$ & 6 & 549 & $1.09 \%$ \\
\hline 8 & 0 & 414 & $0.00 \%$ & 5 & 474 & $1.05 \%$ \\
\hline
\end{tabular}

Table 11: Observed pandemic A/H1N1 positivity in the DataMart system.

\section{Model for the first two waves}

\subsection{Parameterisation and likelihood}

Infections The serial prevalence data (section 1.1.3) inform indirectly the cumulative incidence of infection, i.e. $I A R_{w, a}$. The prevalence of antibodies post-first wave, $\pi_{1, a}$, may be expressed as the sum of the baseline proportion with antibodies, $\pi_{\text {baseline }, a}$ and the cumulative incidence $I A R_{1, a}$ over the first wave:

$$
\pi_{1, a}=\pi_{\text {baseline }, a}+I A R_{1, a}
$$

This prevalence $\pi_{1, a}$ is therefore a functional parameter, whereas $\pi_{b a s e l i n e, a}$ is a basic parameter. The sero-prevalence data post-second wave may be used similarly to inform the attack rate in the second wave, on the basis that:

$$
\pi_{2, a}=\pi_{\text {baseline }, a}+I A R_{1, a}+I A R_{2, a}
$$

where the prevalence $\pi_{2, a}$ is again a functional parameter. However, the main model presented in Presanis et al. (2011) does not include the post-second wave sero-prevalence data, for a few reasons. There are concerns that the serology samples taken during the pandemic might have come from a population at greater risk of infection than in the general population. Individuals in the sample therefore may have been more likely to have been vaccinated in the second wave than the general population, possibly introducing bias (Miller et al., 2010; Hardelid et al., 2011; Bird, 2010). Since vaccination and infection may both lead indistinguishably to sero-positive tests, a greater than expected proportion of individuals with titre greater than 1:32 may have been observed in the study sample. Furthermore, small sample sizes may imply limited power to detect changes in prevalence (Miller et al., 2010; Hardelid et al., 2011). The post-second wave data are therefore only used in sensitivity analyses (see section 6.1 of the main text).

The observed number $y_{1, a}$ of samples positive for pandemic A/H1N1 influenza antibodies post-first wave (Table 4) is Binomially distributed with size given by the number $n_{1, a}$ of samples tested and proportion $\pi_{1, a}$, and similarly for the observed number $y_{0, a}$ at baseline (pre-2009 pandemic):

$$
\begin{aligned}
& y_{1, a} \sim \operatorname{Bin}\left(n_{1, a}, \pi_{1, a}\right) \\
& y_{0, a} \sim \operatorname{Bin}\left(n_{0, a}, \pi_{\text {baseline }, a}\right)
\end{aligned}
$$

If the post-second wave sero-prevalence data were to be used, as in the sensitivity analyses of section 6.1 of the main text, the observed number $y_{2, a}$ of samples testing positive would again be Binomially distributed:

$$
y_{2, a} \sim \operatorname{Bin}\left(n_{2, a}, \pi_{2, a}\right)
$$


Symptomatic infections The weekly HPA symptomatic case estimates $\hat{O}_{t, a, r, S}$ (section 1.1.2), summed over geographic regions $r$ and weeks $t$ within each wave, inform $N_{w, a, S}$, the number of symptomatic cases, though indirectly, as they are thought to be under-estimates. The bias may be due to overly-strong assumptions made in the midst of the pandemic, such as a fixed proportion accessing health-care (Health Protection Agency, 2009; Evans et al., 2011). The belief that a bias exists is expressed by assuming the sum $\hat{O}_{w, a, S}=\sum_{t \in \text { wave } w, r} \hat{O}_{t, a, r, S}$ informs a biased estimate $N_{w, a, S B}$ of the true number of symptomatic infections, $N_{w, a, S}$. We take the $\log$ of the sum $\hat{O}_{w, a, S}$ to be a realisation of the following normal distribution:

$$
\log \left(\hat{O}_{w, a, S}\right) \sim N\left(\log \left(N_{w, a, S B}\right), \sigma_{w, a, S B}^{2}\right)
$$

where $\sigma_{w, a, S B}$ is a fixed standard deviation obtained by assuming the ranges in the HPA case estimates, $\log \left(O_{w, a, S}^{\text {low }}\right)$ and $\log \left(O_{w, a, S}^{\text {high }}\right)$ (section 1.1.2 above), lie three standard deviations away from the central estimate $\log \left(\hat{O}_{w, a, S}\right)$.

Any potential bias is modelled by a detection probability $d_{w, a, S}$, assuming that:

$$
N_{w, a, S B} \sim \operatorname{Bin}\left(N_{w, a, S}, d_{w, a, S}\right)
$$

Hospitalisations The number of confirmed cases in hospital ( $O_{w, a, H}$, section 1.1.4 above) is also known to be under-ascertained, so that

$$
O_{w, a, H} \sim \operatorname{Bin}\left(N_{w, a, H}, d_{w, H}\right)
$$

where $d_{w, H}$ is the detection probability for hospitalisation, assumed equal across age groups. Out of hospitalised individuals for whom we know final outcomes (discharge, transfer or death), the observed numbers leading to ICU-admission $\left(O_{w, a, I}^{W S 1}\right)$ and death $\left(O_{w, a, D}^{W S 2}\right)$ in the same source (iv) are taken to unbiasedly inform the conditional probabilities $c_{w, a, I \mid H}$ and $c_{w, a, D \mid H}$ respectively:

$$
\begin{aligned}
O_{w, a, I}^{W S 1} & \sim \operatorname{Bin}\left(O_{w, a, H}^{W S 1}, c_{w, a, I \mid H}\right) \\
O_{w, a, D}^{W S 2} & \sim \operatorname{Bin}\left(O_{w, a, H}^{W S 2}, c_{w, a, D \mid H}\right)
\end{aligned}
$$

The denominators (numbers of hospitalisations) $O_{w, a, H}^{W S 1}$ and $O_{w, a, H}^{W S 2}$ are not necessarily equal to each other or to the total observed hospitalisations $O_{w, a, H}$, since some individuals were missing data on whether or not they were admitted to ICU or on final outcomes. The notation $W S 1$ and $W S 2$ therefore denotes the sets of hospitalisations for which data on ICU-admission and final outcome respectively were non-missing.

Deaths The reported deaths $O_{w, a, D}$ of section 1.1.5 above, are also under-ascertained so we let

$$
O_{w, a, D} \sim \operatorname{Bin}\left(N_{w, a, D}, d_{w, D}\right)
$$

with detection probability $d_{w, D}$ equal across age groups.

The likelihood function The total likelihood is therefore the product of the contributions from each data source:

$$
\begin{aligned}
L(\boldsymbol{y} \mid \boldsymbol{\Theta}) & =\prod_{a}\left(\begin{array}{c}
n_{1, a} \\
y_{1, a}
\end{array}\right) \pi_{1, a}^{y_{1, a}}\left(1-\pi_{1, a}\right)^{n_{1, a}-y_{1, a}} \prod_{a}\left(\begin{array}{c}
n_{0, a} \\
y_{0, a}
\end{array}\right) \pi_{\text {baseline }, a}^{y_{0, a}}\left(1-\pi_{\text {baseline }, a}\right)^{n_{0, a}-y_{0, a}} \\
& \times \prod_{w, a} \frac{1}{\sqrt{2 \pi \sigma_{w, a, S B}^{2}}} \exp \left\{-\frac{\left(\log \left(\sum_{t \in \text { wave } w, r} \hat{O}_{t, a, r, S}\right)-\log \left(N_{w, a, S B}\right)\right)}{2 \sigma_{w, a, S B}^{2}}\right\} \\
& \times \prod_{w, a}\left(\begin{array}{c}
N_{w, a, H} \\
O_{w, a, H}
\end{array}\right) d_{w, H}^{O_{w, a, H}}\left(1-d_{w, H}\right)^{N_{w, a, H}-O_{w, a, H}} \\
& \times \prod_{w, a}\left(\begin{array}{c}
O_{w, a, H}^{W S 1} \\
O_{w, a, I}^{W S 1}
\end{array}\right) c_{w, a, I \mid H}^{O_{w, I}^{W S 1}}\left(1-c_{w, a, I \mid H}\right)^{O_{w, a, H}^{W S 1}-O_{w, a, I}^{W S 1}} \\
& \times \prod_{w, a}\left(\begin{array}{c}
O_{w, a, H}^{W S 2} \\
O_{w, a, D}^{W S 2}
\end{array}\right) c_{w, a, D \mid H}^{O_{w, D}^{W S 2}}\left(1-c_{w, a, D \mid H}\right)^{O_{w, a, H}^{W S 2}-O_{w, a, D}^{W S 2}} \\
& \times \prod_{w, a}\left(\begin{array}{c}
N_{w, a, D} \\
O_{w, a, D}
\end{array}\right) d_{w, D}^{O_{w, a, D}}\left(1-d_{w, D}\right)^{N_{w, a, D}-O_{w, a, D}}
\end{aligned}
$$




\subsection{Priors}

The full list of prior distributions assigned to each basic parameter is given in Table 12 .

The prior distribution for the detection probability for symptomatic infection, $d_{w, a, S}$, in the first wave is a $\operatorname{Beta}(1,1)$ and the detection probability is identified through the inclusion of the sero-prevalence data in the model, together with an informative prior for the proportion of infections that are symptomatic, $c_{S \mid \text { Inf }}$. Due to a paucity of information on this proportion symptomatic, it is kept constant across age groups and waves, and is assigned a prior with mean $40 \%$ and $95 \%$ prior probability of lying between 30 and $50 \%$, covering a range of estimates in the seasonal influenza literature (Johnson et al., 2011; Carrat et al., 2008; Monto et al., 1985):

$$
c_{S \mid I n f} \sim \operatorname{Beta}(40,60)
$$

As the post-second wave sero-prevalence data have not been included in the model, in order for the number of infections $N_{2, a, I}$ to be identified, an informative prior for the second wave detection probability for the HPA case estimates, $d_{2, a, S}$, is required. This is achieved by assuming $d_{2, a, S}$ to be similar to, but with greater uncertainty, than $d_{1, a, S}$ :

$$
\begin{aligned}
\operatorname{logit}\left(d_{2, a, S}\right) & =\operatorname{logit}\left(d_{1, a, S}\right)+\epsilon_{a} \\
\epsilon_{a} & \sim N\left(0,0.134^{2}\right)
\end{aligned}
$$

The standard deviation of the Normal distribution for $\epsilon_{a}$ is chosen so that the odds $\operatorname{ratio} \exp \left(\epsilon_{a}\right) \operatorname{varies}$ between 0.77 and 1.30 .

Each conditional probability of hospitalisation given symptomatic infection, $s C H R_{w, a}=c_{w, a, H \mid S}$, is given an informative Uniform prior distribution independently for each $(w, a)$ pair:

$$
{ }_{s C H R} R_{w, a} \sim \operatorname{Unif}\left[0, c C H R_{a}\right]
$$

with upper limit $c C H R_{a}$, the corresponding age-specific confirmed case-hospitalisation risk. These $c C H R_{a}$ are themselves given age-specific informative Beta prior distributions reflecting the estimates of section 1.1.1 of the appendix (Table 1), with a mean over age groups of $1.20 \%$ (95\% CI: 0.97-1.49\%)).

The detection probability for death, $d_{w, D}$, is given an informative $\operatorname{Beta}(45,5)$ prior independently in each wave $w=1,2$, reflecting estimates of $90 \%$ (95\% CI: $80-100 \%)$ from a capture-recapture study (Grant, 2010; Presanis et al., 2011).

\begin{tabular}{ll}
\hline Parameter & Prior \\
\hline$\left(I A R_{1, a}, I A R_{2, a}, 1-\sum_{w=1}^{2} I A R_{w, a}\right)$ & $\operatorname{Dirichlet}(1,1,1)$ \\
$\pi_{\text {baseline }, a}$ & $\operatorname{Beta}(1,1)$ \\
$d_{1, a, S}$ & $\operatorname{Beta}(1,1)$ \\
$\epsilon_{a}$ & $\operatorname{Normal}\left(0,0.134^{2}\right)$ \\
$d_{w, H}$ & $\operatorname{Beta}(1,1)$ \\
$s C H R_{w, a}$ & $\operatorname{Unif}\left[0, c C H R_{a}\right]$ \\
$c C H R_{1}$ & $\operatorname{Beta}(6.6,61.5)$ \\
$c C H R_{2}$ & $\operatorname{Beta}(1.4,123.0)$ \\
$c C H R_{3}$ & $\operatorname{Beta}(12.8,1929.6)$ \\
$c C H R_{4}$ & $\operatorname{Beta}(13.3,1097.6)$ \\
$c C H R_{5}$ & $\operatorname{Beta}(13.0,851.1)$ \\
$c C H R_{6}$ & $\operatorname{Beta}(2.0,168.4)$ \\
$c C H R_{7}$ & $\operatorname{Beta}(1.7,17.9)$ \\
$d_{w, D}$ & $\operatorname{Beta}(45,5)$ \\
\hline
\end{tabular}

Table 12: Prior distributions for the set of basic parameters.

\section{Model for the third wave}

\subsection{Parameterisation and likelihood}

Infections There were no post-third wave sero-prevalence data available at time of analysis, but the attack rate $I A R_{3, a}$ is still identifiable given the data and informative prior distributions in the rest of the 
model.

Symptomatic infections To estimate the number of symptomatic pandemic A/H1N1 infections in the third wave, a fully Bayesian regression model fitted jointly to the RCGP ILI consultation and RCGP/RMN virological datasets (section 1.2.1) is employed. A similar regression model was used in Birrell et al. (2011) to estimate the week- and age-specific "background" consultation rates, i.e. those ILI consultations not due to the pandemic A/H1N1 strain. Here the complementary pandemic consultation rate is required. This regression model improves on the HPA method of Section 1.1.2 in two key respects: the Bayesian joint modelling of both datasets ensures uncertainty is fully and correctly propagated from the data to the estimates; and the model formally incorporates an informative prior distribution for the proportion consulting a general practitioner (GP), based on previous work (Birrell et al., 2011).

The observed weekly and age-specific counts of ILI consultations, $y_{t, a}^{G P}$, are assumed to be realisations of a negative binomial likelihood, to account for over-dispersion in the data:

$$
y_{t, a}^{G P} \sim N B\left(\delta, \frac{\mu_{t, a}}{\mu_{t, a}+\delta}\right)
$$

where the dispersion parameter is $\delta$, the expected number of ILI consultations is $\mu_{t, a}$ and the variance is $\mu_{t, a}+\mu_{t, a}^{2} / \delta$.

Given the small sample sizes in the RCGP/RMN positivity data (Figure 2), the three youngest age groups are collapsed into a single $<15$ age group, so that the positivity data are indexed by $b \in\{<$ $15,15-24,25-44,45-64,65+\}$. The numbers of virologically positive tests $y_{t, b}^{R}$ are binomial realisations, assuming that the tests have specificity equal to 1 and (fixed) sensitivity $s=0.8$ :

$$
y_{t, b}^{R} \sim \operatorname{Bin}\left(n_{t, b}^{R}, s \times p_{t, b}^{R}\right)
$$

The expected positivity $p_{t, b}^{R}$ is then regressed logistically on time $t$ and age $b$, while the expected ILI consultation rate per 100,000 population, $\mu_{t, a} /\left(100,000 \times n_{t, a}^{G P}\right)$, is regressed log-linearly on time $t$ and age $a$. The pandemic A/H1N1 GP consultation rate per 100,000 population is then estimated as $h_{t, a}=p_{t, b_{a}}^{R} \times \mu_{t, a} /\left(100,000 \times n_{t, a}^{G P}\right)$, where $b_{a}=a$ if $a$ is one of the $\geq 15$ age groups and is $<15$ otherwise. Finally, the cumulative number of symptomatic pandemic A/H1N1 cases over the third wave is estimated as

$$
O_{3, a, S}=\sum_{t \in \text { wave } 3} h_{t, a} / p_{G P, c_{a}}
$$

where the child or adult proportion of symptomatic individuals consulting a GP, $p_{G P, c_{a}}$ for $c_{a} \in\{<15, \geq$ $15\}$ depending on age group $a$, is given an informative prior based on the posterior estimate from Birrell et al. (2011).

Samples from the joint posterior distribution from the above joint regression model were obtained by MCMC simulation, using JAGS (Plummer, 2010). Results shown here are for the full RCGP/RMN positivity data. Plots comparing visually the observed and the posterior predictive distribution of the positivity and the ILI consultation rate are shown respectively in Figures 3 and 4. The posterior median and $95 \%$ credible interval of the number symptomatic $O_{3, a, S}$ in each age group are given in Table 13 .

\begin{tabular}{rrrr}
\hline Age & Median & \multicolumn{2}{c}{$95 \%$ CI } \\
\hline$<1$ & 16,732 & 8,976 & 29,923 \\
$1-4$ & 136,435 & 79,147 & 229,643 \\
$5-14$ & 253,106 & 147,302 & 420,530 \\
$15-24$ & 418,284 & 240,672 & 713,943 \\
$25-44$ & 915,173 & 326,577 & $1,536,511$ \\
$45-64$ & 570,479 & 159,332 & 961,576 \\
$65+$ & 30,296 & 13,883 & 62,285 \\
\hline
\end{tabular}

Table 13: Posterior median and 95\% credible interval of the number symptomatic $O_{3, a, S}$ from the joint regression model of primary care consultation and virological positivity.

The estimates of Table 13 are thought to be unbiased estimates of $N_{3, a, S}$, so in the main severity model, we assume a detection probability equal to 1 . To ensure $N_{3, a, S}$ lies on the positive real line, 

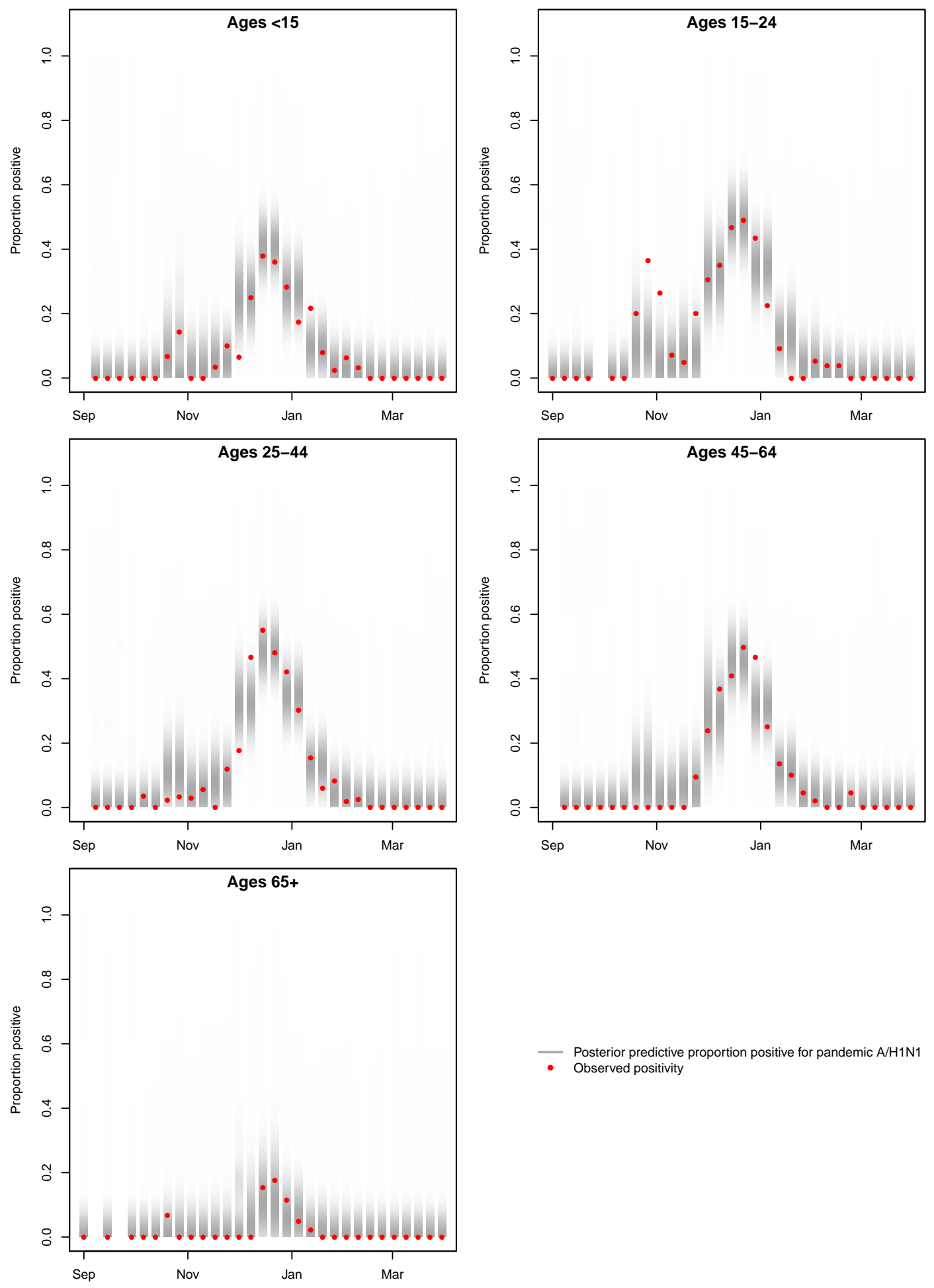

- Posterior predictive proportion positive for pandemic $\mathrm{A} / \mathrm{H} 1 \mathrm{~N} 1$ - Observed positivity

Figure 3: Observed versus posterior predictive positivity for pandemic A/H1N1 (unadjusted for test sensitivity), by age group and week, in the period September 2010 to March 2011. 

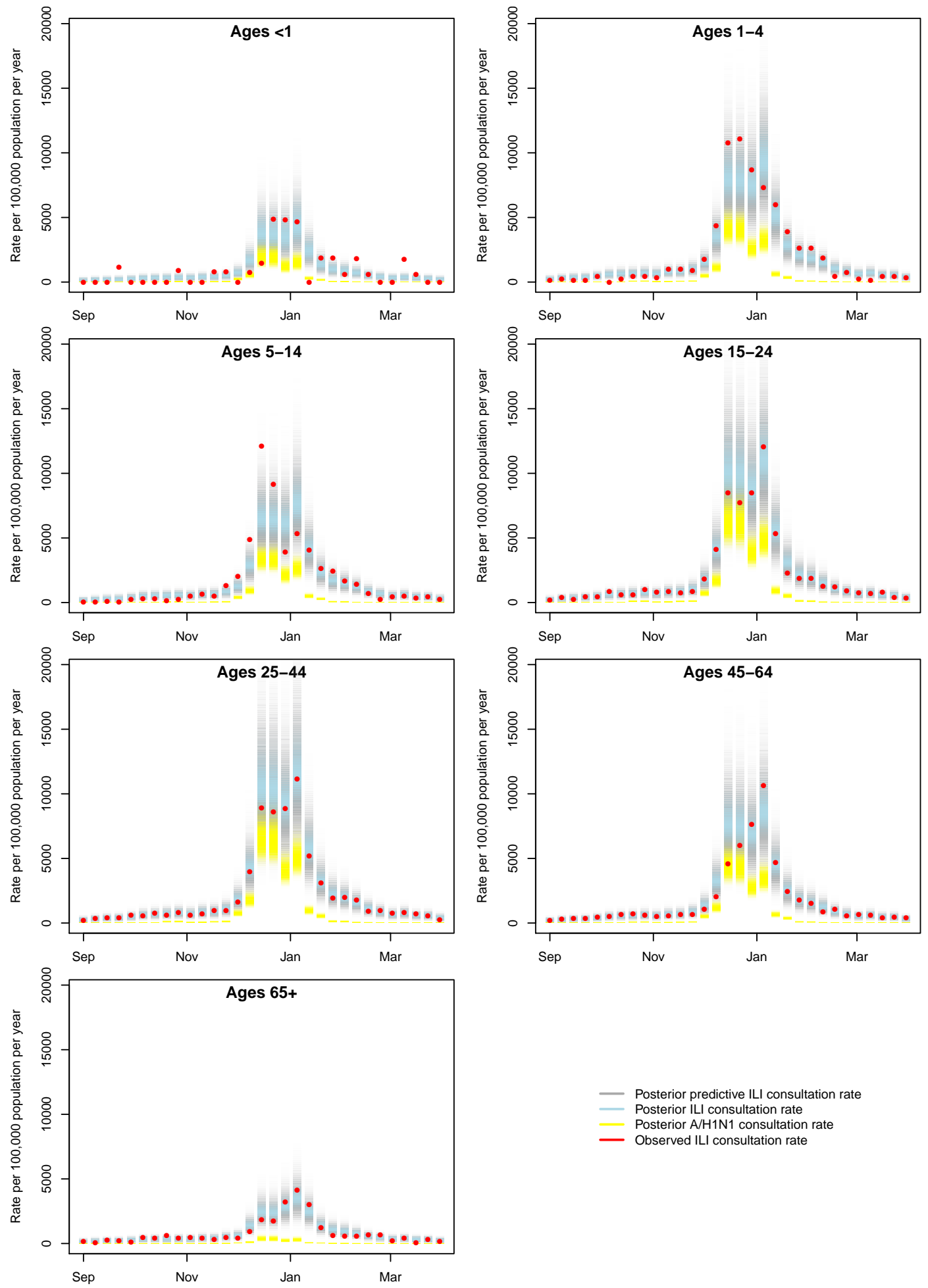

Figure 4: Observed versus posterior GP consultation rates, by age group and week, in the period September 2010 to March 2011. Total ILI consultation rates are shown in grey (posterior predictive distribution) and light blue (posterior distribution). The posterior pandemic A/H1N1 consultation rate is shown in yellow. 
we obtain from the regression model the posterior mean $\left(\hat{O}_{3, a, S}^{\prime}\right)$ and standard deviation $\left(\sigma_{3, a, S}^{\prime}\right)$ of the logarithm of the number symptomatic, and assume $O_{3, a, S}^{\prime}$ is a realisation of a normal distribution:

$$
\hat{O}_{3, a, S}^{\prime} \sim N\left(\log \left(N_{3, a, S}\right), \sigma_{3, a, S}^{\prime 2}\right)
$$

A sensitivity analysis restricting the virological positivity data to samples from the RCGP scheme for whom we know dates of both ILI consultation and swabbing, and where the swabs were taken within 5 days of the ILI consultation, yields results from the joint regression model as given in Table 14. Note

\begin{tabular}{rrrr}
\hline Age & Median & \multicolumn{2}{c}{$95 \%$ CI } \\
\hline$<1$ & 22,316 & 11,812 & 41,174 \\
$1-4$ & 186,450 & 106,118 & 320,960 \\
$5-14$ & 347,675 & 198,815 & 596,655 \\
$15-24$ & 424,764 & 240,084 & 733,978 \\
$25-44$ & $1,065,977$ & 615,853 & $1,816,424$ \\
$45-64$ & 648,197 & 368,010 & $1,125,323$ \\
$65+$ & 21,186 & 4,990 & 62,610 \\
\hline
\end{tabular}

Table 14: Posterior median and 95\% credible interval of the number symptomatic $O_{3, a, S}$ from the joint regression model of primary care consultation and virological positivity, where the positivity data are restricted to those swabs taken within 5 days of an ILI consultation.

the higher positivity observed by restricting the samples results in higher estimates of the number of symptomatic infections, although the credible intervals in the two sets of estimates overlap considerably. The estimates of Table 13 are used in the main results of Section 6 of the main text, but the estimates of Table 14 are also used in a sensitivity analysis in Section 6.1 of the main text.

Hospital admissions The USISS surveillance scheme (section 1.2.2) provides data on a lower bound for the cumulative number of hospital admissions of confirmed cases $N_{3, b, H}^{*}$ in five age groups $b \in\{<$ $5,5-14,15-44,45-64,65+\}$, so we take the observed data to be realisations of a binomial distribution

$$
O_{3, b, H} \sim \operatorname{Bin}\left(N_{3, b, H}^{*}, d_{3, H}\right)
$$

where the $N_{3, b, H}^{*}$ are sums over age groups:

$$
\begin{aligned}
N_{3,<5, H}^{*} & =N_{3,<1, H}+N_{3,1-4, H} \\
N_{3,15-44, H}^{*} & =N_{3,15-24, H}+N_{3,25-44, H} \\
N_{3, b, H}^{*} & =N_{3, b, H}, \quad b \in\{5-14,45-64,65+\} .
\end{aligned}
$$

Additionally, USISS provides information on the proportion of observed hospital admissions that result in ICU admission, realisations of a binomial distribution

$$
O_{3, b, I} \sim \operatorname{Bin}\left(O_{3, b, H}, c_{3, b, I \mid H}^{*}\right)
$$

where the $c_{3, b, I \mid H}^{*}$ are weighted averages of the conditional probabilities of ICU admission given hospitalisation in the appropriate age groups:

$$
\begin{aligned}
c_{3,<5, I \mid H}^{*} & =\frac{N_{3,<1, H} c_{3,<1, I \mid H}+N_{3,1-4, H} c_{3,1-4, I \mid H}}{N_{3,<1, H}+N_{3,1-4, H}} \\
c_{3,15-44, I \mid H}^{*} & =\frac{N_{3,15-24, H} c_{3,15-24, I \mid H}+N_{3,25-44, H} c_{3,25-44, I \mid H}}{N_{3,15-24, H}+N_{3,25-44, H}} \\
c_{3, b, I \mid H}^{*} & =c_{3, b, I \mid H}, \quad b \in\{5-14,45-64,65+\} .
\end{aligned}
$$

ICU admissions As the USISS hospitalisation and ICU data provide small sample sizes, we additionally use independent data on the number of suspected cases occupying ICU each week (Table 10 of section 1.2.3), i.e. prevalence-type data, from which it is possible to estimate the cumulative number of 
admissions of confirmed cases. This is achieved by fitting an immigration-death process model to the prevalence-type data on ICU occupancy (Department of Health, 2011) and to virological positivity data (section 1.2.3) using also prior information, from the hospitalisation data of the first two waves, on the distribution of length of stay in ICU (see section 4 for details).

The immigration-death process model results in estimates (posterior mean (sd)) of the cumulative number of new admissions of confirmed cases, $\hat{O}_{\text {child,I }}\left(\sigma_{\text {child,I }}\right)$ in children and $\hat{O}_{\text {adult }, I}\left(\sigma_{\text {adult }, I}\right)$ in adults. Denote the posterior mean (sd) of the logarithm of the cumulative number of new admissions of confirmed cases by $\hat{O}_{b, I}^{\prime}\left(\sigma_{b, I}^{\prime}\right)$ for the two age groups $b=$ child and $b=$ adult. We assume they are drawn from Normal distributions:

$$
\hat{O}_{b, I}^{\prime} \sim N\left(\log \left(N_{b, I}^{*}\right), \sigma_{b, I}^{\prime 2}\right)
$$

where $N_{\text {child,I }}^{*}$ and $N_{\text {adult }, I}^{*}$ are both sums over age groups $a$ in the severity model:

$$
\begin{aligned}
& N_{\text {child }, I}^{*}=N_{<1, I}^{*}+N_{1-4, I}^{*}+N_{5-14, I}^{*} \\
& N_{\text {adult }, I}^{*}=N_{15-24, I}^{*}+N_{25-44, I}^{*}+N_{45-64, I}^{*}+N_{65+, I}^{*}
\end{aligned}
$$

And finally, $N_{a, I}^{*}$ in each of the 7 age groups $a$ is assumed to be an under-ascertained version of the true cumulative number of confirmed cases in ICU, $N_{3, a, I}$ :

$$
N_{a, I}^{*} \sim \operatorname{Bin}\left(N_{3, a, I}, d_{3, I}\right) \quad \text { for each age group } a
$$

where $d_{3, I}$ is a detection probability, since the data on suspected cases only cover a proportion of the time period we are considering.

Deaths The observed deaths in the third wave (section 1.1.5) are again assumed to represent only a proportion $d_{3, D}$ of all deaths in the third wave:

$$
O_{3, a, D} \sim \operatorname{Bin}\left(N_{3, a, D}, d_{3, D}\right)
$$

The likelihood function The total likelihood for the third wave $(w=3)$ analysis is therefore the product of the contributions from each data source:

$$
\begin{aligned}
L(\boldsymbol{y} \mid \boldsymbol{\Theta}) & =\prod_{w, a} \frac{1}{\sqrt{2 \pi \sigma_{w, a, S}^{\prime 2}}} \exp \left\{-\frac{\left(O_{w, a, S}^{\prime}-\log \left(N_{w, a, S}\right)\right)}{2 \sigma_{w, a, S}^{\prime 2}}\right\} \\
& \times \\
& \times \prod_{w, b \in\{<5,5-14,15-44,45-64,65+\}}\left(\begin{array}{l}
N_{w, b, H}^{*} \\
O_{w, b, H}
\end{array}\right) d_{w, H}^{O_{w, b, H}}\left(1-d_{w, H}\right)^{N_{w, b, H}^{*}-O_{w, b, H}} \\
& \times \prod_{w, b \in\{<5,5-14,15-44,45-64,65+\}}\left(\begin{array}{c}
O_{w, b, H} \\
O_{w, b, I}
\end{array}\right)\left(c_{w, b, I \mid H}^{*}\right)^{O_{w, b, I}}\left(1-c_{w, b, I \mid H}^{*}\right)^{O_{w, b, H}-O_{w, b, I}} \\
& \times \prod_{w, b \in\{c h i l d, a d u l t\}} \frac{1}{\sqrt{2 \pi \sigma_{b, I}^{\prime 2}}} \exp \left\{-\frac{\left(\hat{O}_{b, I}^{\prime}-\log \left(N_{b, I}^{*}\right)\right)}{2 \sigma_{b, I}^{\prime 2}}\right\} \\
& \prod_{w, a}\left(\begin{array}{c}
N_{w, a, D} \\
O_{w, a, D}
\end{array}\right) d_{w, D}^{O_{w, a, D}}\left(1-d_{w, D}\right)^{N_{w, a, D}-O_{w, a, D}}
\end{aligned}
$$

\subsection{Priors}

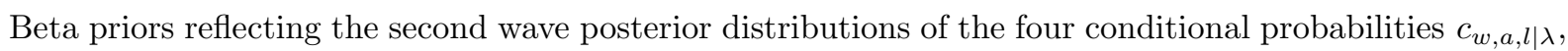
for each $(l, \lambda) \in\{S|\operatorname{Inf}, H| S, I|H, D| H\}$ are given to the corresponding third wave probabilities. For the proportion symptomatic, which is not age-specific, this prior is $c_{3, S \mid I n f} \sim \operatorname{Beta}(37,69)$. The other priors are specified in Table 15.

The detection probability for hospitalisation, $d_{w, H}$ is assigned a Beta $(1,1)$ prior, while for ICU admission, $d_{w, I}$, is given a mildly informative $\operatorname{Beta}(9,6)$ prior reflecting the fact that we believe the probability is less than 1 but greater than the detection probability for hospitalisation estimated in the first two waves (around $20-30 \%$ ). However, as this system is a different surveillance system to that used in the 
first two waves, we leave the prior relatively diffuse, with a prior mean of $60 \%$ and $95 \%$ prior mass in $35-82 \%$. We assume the detection probability for death, $d_{w, D}$, in the third wave was less than in the second wave, by employing a Uniform prior distribution:

$$
d_{3, D} \sim \operatorname{Unif}\left[0, d_{2, D}\right]
$$

\begin{tabular}{|c|c|c|c|c|c|c|c|c|}
\hline \multirow{2}{*}{$\begin{array}{l}\text { Parameter } \\
\text { Prior } \\
\text { Age } \\
\end{array}$} & \multicolumn{2}{|c|}{$\begin{array}{l}\left(I A R_{w, a}\right) \\
\operatorname{Dirichlet}\left(2 x_{a} / y_{a}, 1,1\right)\end{array}$} & \multicolumn{2}{|c|}{$\begin{array}{l}c_{3, a, H \mid S} \\
\operatorname{Beta}\left(x_{a}, y_{a}\right)\end{array}$} & \multicolumn{2}{|c|}{$\begin{array}{l}c_{3, a, I \mid H} \\
\operatorname{Beta}\left(x_{a}, y_{a}\right)\end{array}$} & \multicolumn{2}{|c|}{$\begin{array}{l}c_{3, a, D \mid H} \\
\operatorname{Beta}\left(x_{a}, y_{a}\right)\end{array}$} \\
\hline & $x_{a}$ & $y_{a}$ & $x_{a}$ & $y_{a}$ & $x_{a}$ & $y_{a}$ & $x_{a}$ & $y_{a}$ \\
\hline$<1$ & 6 & 31 & 4 & 91 & & 76 & 30 & 2,501 \\
\hline $1-4$ & 5 & 16 & 4 & 372 & & 132 & 43 & 2,834 \\
\hline $5-14$ & 9 & 17 & & 1,924 & 16 & 179 & 53 & 2,364 \\
\hline $15-24$ & 35 & 109 & 10 & 1,292 & 16 & 119 & 51 & 1,827 \\
\hline $25-44$ & 18 & 80 & 6 & 755 & 50 & 152 & 62 & 1,190 \\
\hline $45-64$ & 20 & 103 & 3 & 406 & 56 & 83 & 60 & 553 \\
\hline $65+$ & 126 & 445 & 1 & 57 & 13 & 29 & 38 & 193 \\
\hline
\end{tabular}

Table 15: Prior distributions for the third wave model.

\section{Estimating cumulative confirmed ICU cases}

Since the data of section 1.2.3 do not directly inform the cumulative number of confirmed cases admitted to ICU with pandemic influenza infection over the third wave, a model is required to estimate this quantity. This model is described in section 4.2, but first we describe estimation of the length of stay in ICU, which is required as an input to the model of section 4.2.

\subsection{Length of stay in ICU}

We estimated the distribution of length of stay in ICU (time from admission to either discharge, transfer or death) by fitting an exponential model to the individual-level data from the 2009-10 web-based hospitalisation data of the first two waves (section 1.1.4). These data include information on date of admittance to ICU, final outcome (discharge, transfer or death) and date of final outcome. Individuals who were missing data on their final outcome or date of final outcome were censored at 0.5 days after time of admission to ICU. The parametric survival model included age as a categorical covariate (Children $b<15$ years, Adults $b \geq 15$ years, not known $b=N K)$. A Weibull distribution was also considered, but the scale parameter was not significantly different from 1 , indicating the exponential model was adequate. Results are shown in Figures 5 and 6.

The parameter estimates for the exponential model with constant hazard $\lambda=\beta_{\text {Adults }}+\beta_{\text {Children }} \mathbb{I}\{b<$ $15\}+\beta_{N K} \mathbb{I}\{b=N K\}$ are given in Table 16. The rate parameter for the exponential distribution for

\begin{tabular}{crrrr}
\hline Parameter & Point estimate & Standard error & z-score & p-value \\
\hline$\beta_{\text {Adults }}$ & 2.7058 & 0.0788 & 34.3324 & $<0.0001$ \\
$\beta_{\text {Children }}$ & -0.4696 & 0.2048 & -2.2936 & 0.0218 \\
$\beta_{N K}$ & 0.0133 & 0.4158 & 0.0321 & 0.9740 \\
\hline
\end{tabular}

Table 16: Parameter estimates for an exponential model of length of stay in ICU.

adults is therefore $\hat{\mu}_{\text {Adults }}=\exp \left(-\hat{\beta}_{\text {Adults }}\right)=\exp (-2.7058)=0.06682$ and for children is $\hat{\mu}_{\text {Children }}=$ $\exp \left(-\hat{\beta}_{\text {Children }}\right)=\exp (-2.7058+0.4696)=0.1069$. These give mean lengths of stay of 14.97 and 9.36 days for adults and children respectively, with standard deviation equal to the mean for the exponential distribution. 


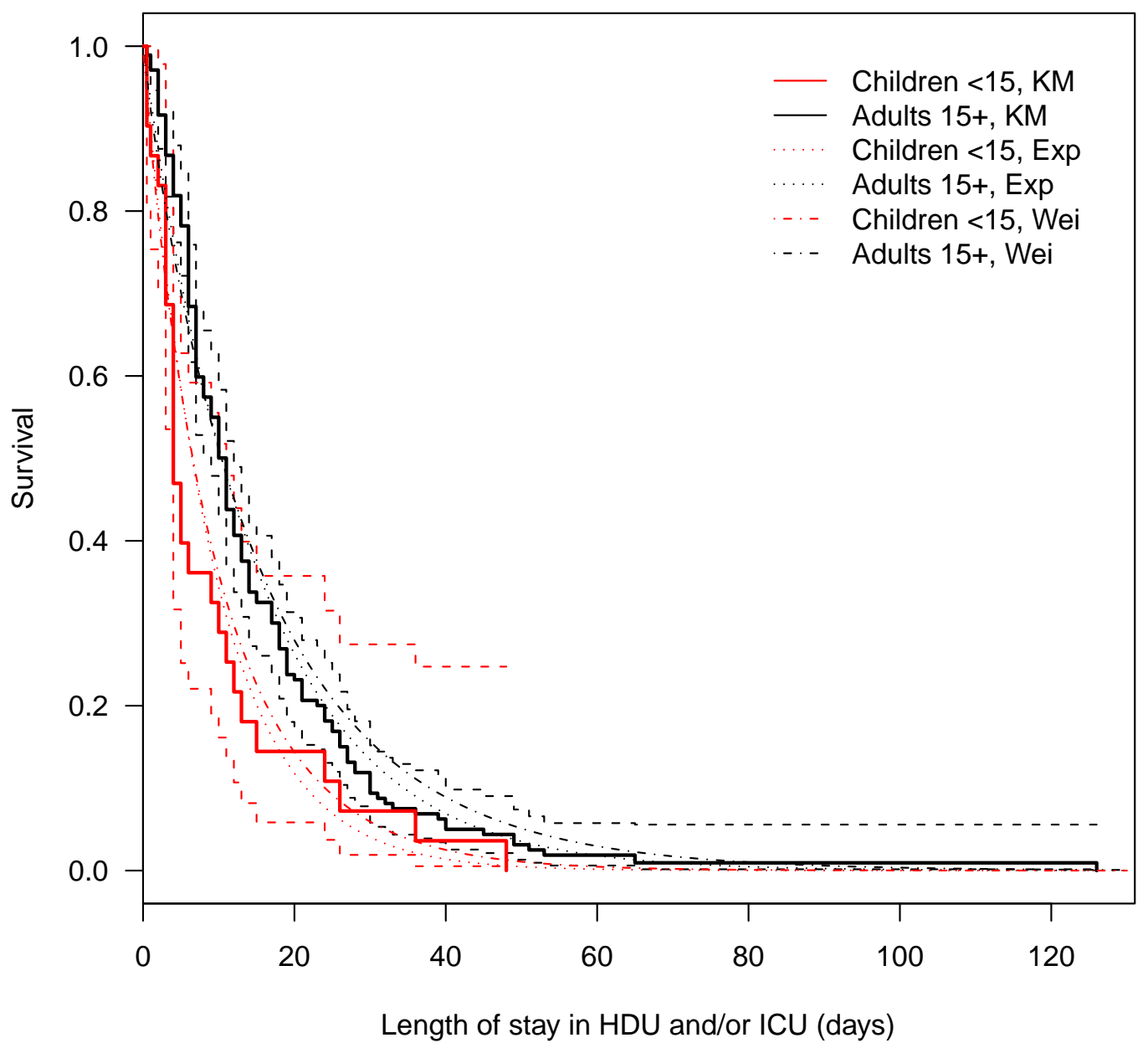

Figure 5: Kaplan-Meier curves for children (red) and adults (black) against the Weibull and Exponential parametric modelled survival.

\subsection{The ICU model}

To estimate cumulative confirmed cases from the data of section 1.2.3, we estimate weekly new admissions of suspected cases, by fitting an immigration-death process to the prevalence-type data (Table 10). We also estimate the corresponding weekly virological positivity for pandemic A/H1N1 influenza from the data in the DataMart system (Table 11). The estimated weekly number of new admissions of suspected cases is then multiplied by the corresponding weekly positivity to obtain the estimated weekly number of new admissions of confirmed cases. Finally, these are summed to obtain the cumulative number of admissions to ICU of confirmed cases.

To estimate weekly new admissions of suspected cases, we consider the compartmental model of Figure 7. The figure displays a bivariate immigration-death process, where "arrivals" are admissions to ICU with suspected pandemic A/H1N1 influenza and "deaths" are discharges, deaths or transfers from ICU. Let $A_{t, b}$ be the number of new admissions for age group $b$ occurring in a short time interval $[t, t+\delta t)$. The sequence over time of new admissions are assumed to occur according to a non-homogeneous Poisson process with rate $\lambda_{t, b}$ at time $t$, so that the expected number of new admissions in $[t, t+\delta t)$ is $\mathbb{E}\left(A_{t, b}\right)=\lambda_{t, b}$. The length of stay $\left(L o S_{b}\right)$ in ICU is considered exponentially distributed with rate $\mu_{b}$.

The process of arrivals between day 0 and day $t$ who will still be present in ICU at time $t$ is a thinned non-homogeneous Poisson process with rate $\lambda_{s, b} \exp \left\{-\mu_{b}(t-s+1)\right\}$ for arrivals at time $s$. The number of prevalent patients in ICU is hence Poisson distributed with expectation

$$
\mathbb{E}\left(I C U_{t, b}\right)=\int_{s=0}^{t} \lambda_{s, b} \exp \left\{-\mu_{b}(t-s)\right\} d s .
$$



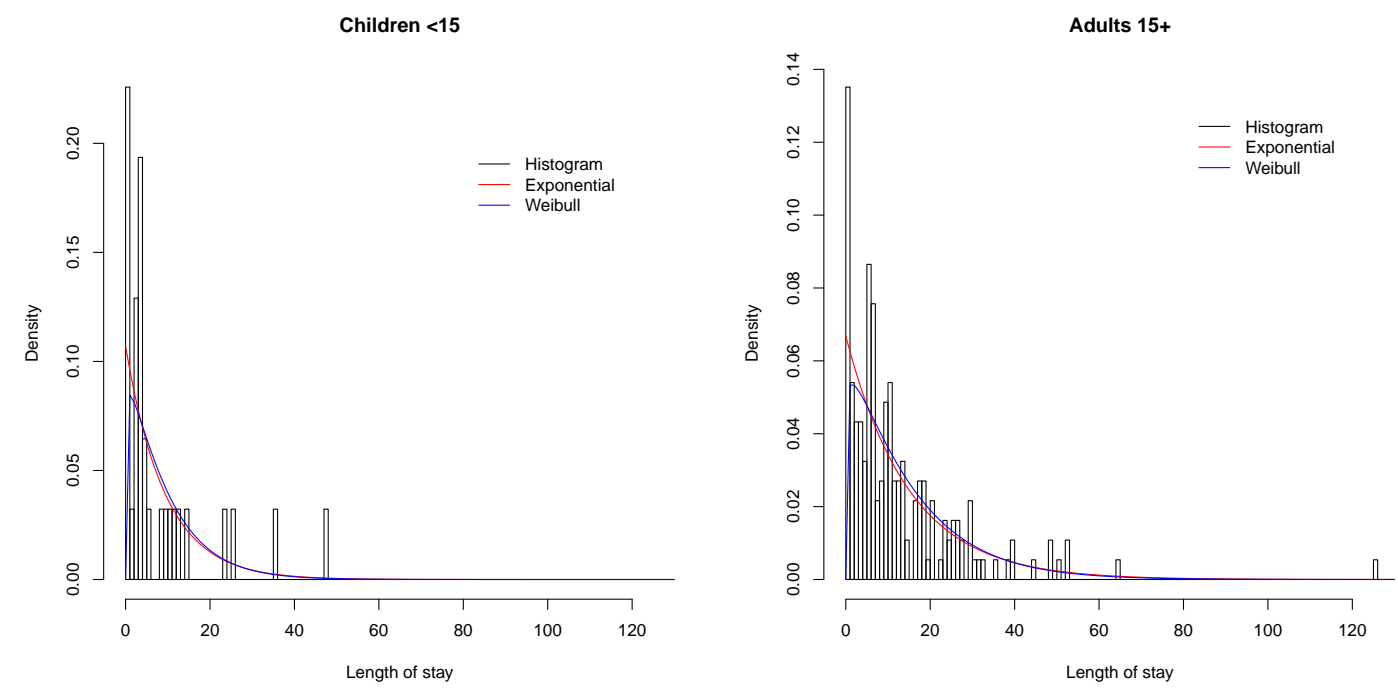

Figure 6: Length of stay distribution: empirical (histogram); Weibull (black line); Exponential (red line).

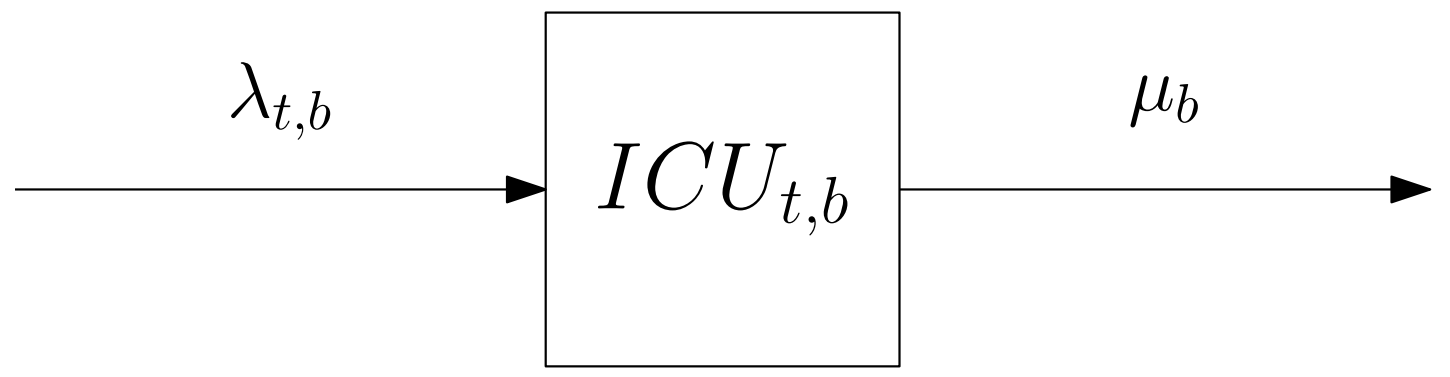

Figure 7: Single state model with "arrivals" at age- and time-specific rate $\lambda_{t, b}$ and "deaths" at age-specific rate $\mu_{b}$.

We employ a discrete time version of the model, with time steps of one day. The expected number of patients prevalent in the ICU state on day $t$, i.e. in $[t, t+1)$ is given by a convolution equation that is the discrete-time version of equation (1):

$$
\mathbb{E}\left(I C U_{t, b}\right)=\sum_{s=1}^{t} \lambda_{s, b} f_{b, s, t}
$$

where $\lambda_{s, b}=\mathbb{E}\left(A_{s, b}\right)$ is the expected number of new admissions on day $s$ and $f_{b, s, t}$ is the probability that a patient admitted on day $s$ is still in the ICU on day $t$, i.e. the probability that the patient's length of stay in ICU is greater than $t-s$ days:

$$
\begin{aligned}
f_{b, s, t} & =\operatorname{Pr}\left\{L o S_{b}>t-s\right\} \\
& =1-\operatorname{Pr}\left\{L o S_{b} \leq t-s\right\} \\
& =1-\left(1-\exp \left\{-\mu_{b}(t-s)\right\}\right) \\
& =\exp \left\{-\mu_{b}(t-s)\right\} .
\end{aligned}
$$

The expected length of stay is $\mathbb{E}\left(L o S_{b}\right)=1 / \mu_{b}$. At the start of day 1 , there are 0 patients present in ICU.

\subsubsection{Priors and likelihood}

We assume the distribution of ICU length of stay is constant over time, and that it is given by the exponential length of stay distribution estimated from individual-level data on hospitalisation during the first two waves (Section 4.1). We therefore take $\mu_{b}=\exp \left\{-\beta_{b}\right\}$ where

$$
\beta_{b} \sim N\left(\hat{\beta}_{b}, \sigma_{b}^{2}\right)
$$


i.e. has prior mean given by the estimate $\hat{\beta}_{b}$ of Section 4.1 with standard deviation given by the corresponding standard error.

The expected number of patients present in ICU on day $1, \mathbb{E}\left(I C U_{1, b}\right)$, is given a Normal prior on the $\log$-scale and we consider a random walk prior for $\log ($ new admissions):

$$
\begin{aligned}
& \log \left(\lambda_{1, b}\right) \sim N\left(0,1000^{2}\right) \\
& \log \left(\lambda_{t, b}\right) \sim N\left(\log \left(\lambda_{t-1, b}\right), \sigma_{R W, b}^{2}\right), \quad t>1
\end{aligned}
$$

The random walk precision, $\gamma_{R W, b}=1 / \sigma_{R W, b}^{2}$, is assumed age group-specific, and is given the same vague gamma prior in each age group $b$ :

$$
\gamma_{R W, b} \sim \Gamma(0.01,0.01)
$$

This prior distribution has mean 1 and variance 100 , so that a $95 \%$ prior interval for the precision is $\left[3.5 \times 10^{-159}, 4.714\right]$. If $\gamma_{R W, b}=1$, then $\sigma_{R W}=1$, implying that the ratio $\lambda_{t, b} / \lambda_{t-1, b}$ lies between 0.14 and 7 for each $t>1$. If $\gamma_{R W, b}=4.714$, this ratio lies between 0.41 and 2.47 , whereas $\gamma_{R W, b}=3.5 \times 10^{-159}$ effectively implies any value for the ratio greater than 0 .

We assume that day 1 , the time at which there are 0 patients with pandemic A/H1N1 influenza infection in the ICU, is the 8th December 2010, which is one week before observations of the number of patients in ICU with suspected A/H1N1 infection began. The observed patients in ICU of Table 10, $y_{t, b, I}$, are Poisson-distributed counts with mean $I C U_{t, b}$ for each $t \in\{8,15,22,29,36,43,50,57,64,71,78\}$.

From these priors and likelihood functions, the posterior distribution of the cumulative number, $\sum_{t} \lambda_{b, t}$, of new admissions for suspected cases of pandemic A/H1N1 influenza may be obtained. By assuming the positivity data from DataMart (Table 11) provide a lower bound for positivity in these suspected cases, we can obtain the posterior distribution of the cumulative number $O_{b, I}$ of confirmed new admissions:

$$
O_{b, I}=\sum_{t} p_{t, b} \lambda_{t, b}
$$

where $p_{t, b} \sim \operatorname{Unif}\left[p_{t, b}^{\text {low }}, 1\right]$ is the positivity and $p_{t, b}^{\text {low }}$ is the lower bound informed by assuming the DataMart data are binomially distributed:

$$
y_{t, b, t}^{D M} \sim \operatorname{Bin}\left(n_{t, b}^{D M}, p_{t, b}^{l o w}\right)
$$

\subsubsection{Computation}

Samples were obtained from the posterior distribution by Markov chain Monte Carlo simulation, using JAGS (Plummer, 2010). Two independent chains were run for 600,000 iterations each, with the first 400,000 iterations discarded as a burn-in period. Convergence of the chains was established by both visual inspection of the trace plots and examination of the Brooks-Gelman-Rubin diagnostic plots (Brooks and Gelman, 1998).

\subsection{Results of the ICU model}

Figure 8 and the low posterior mean deviances in Table 17 shows the fit to the observed prevalent ICU suspected cases to be good. We clearly learn about the random walk precisions, as the prior and posterior distributions are relatively distinct (Figure 9). The posterior mean precisions are somewhat larger than the prior means, at 9.27 for children and 4.89 for adults, implying random walk standard deviations of around 0.33 and 0.45 respectively. These imply that on any given day, the ratio of the number of new admissions to the corresponding number on the previous day is between 0.5 and 1.9 for children and between 0.4 and 2.4 for adults.

These random walk precisions result in posterior distributions of the daily number of new admissions of suspected cases as seen in Figure 10. Given the posterior uncertainty in the random walk precisions, the uncertainty seen in Figure 10 is relatively large, yet there is still a distinct "epidemic curve" seen in both age groups. Furthermore, Figure 11 demonstrates that the cumulative number of new admissions of suspected and confirmed cases are relatively well-identified. For children, we estimate a posterior mean (sd) of 204 (48) suspected and 128 (37) confirmed cases. For adults, these estimates are 1,889 (145) and 1,291 (199) respectively. Denote the posterior mean (sd) of the cumulative confirmed cases by $\hat{O}_{\text {child,I }}\left(\sigma_{\text {child,I }}\right)$ and $\hat{O}_{\text {adult,I }}\left(\sigma_{\text {adult }, I}\right)$ respectively, for use in the third wave severity model of the main text and section 3 above. 

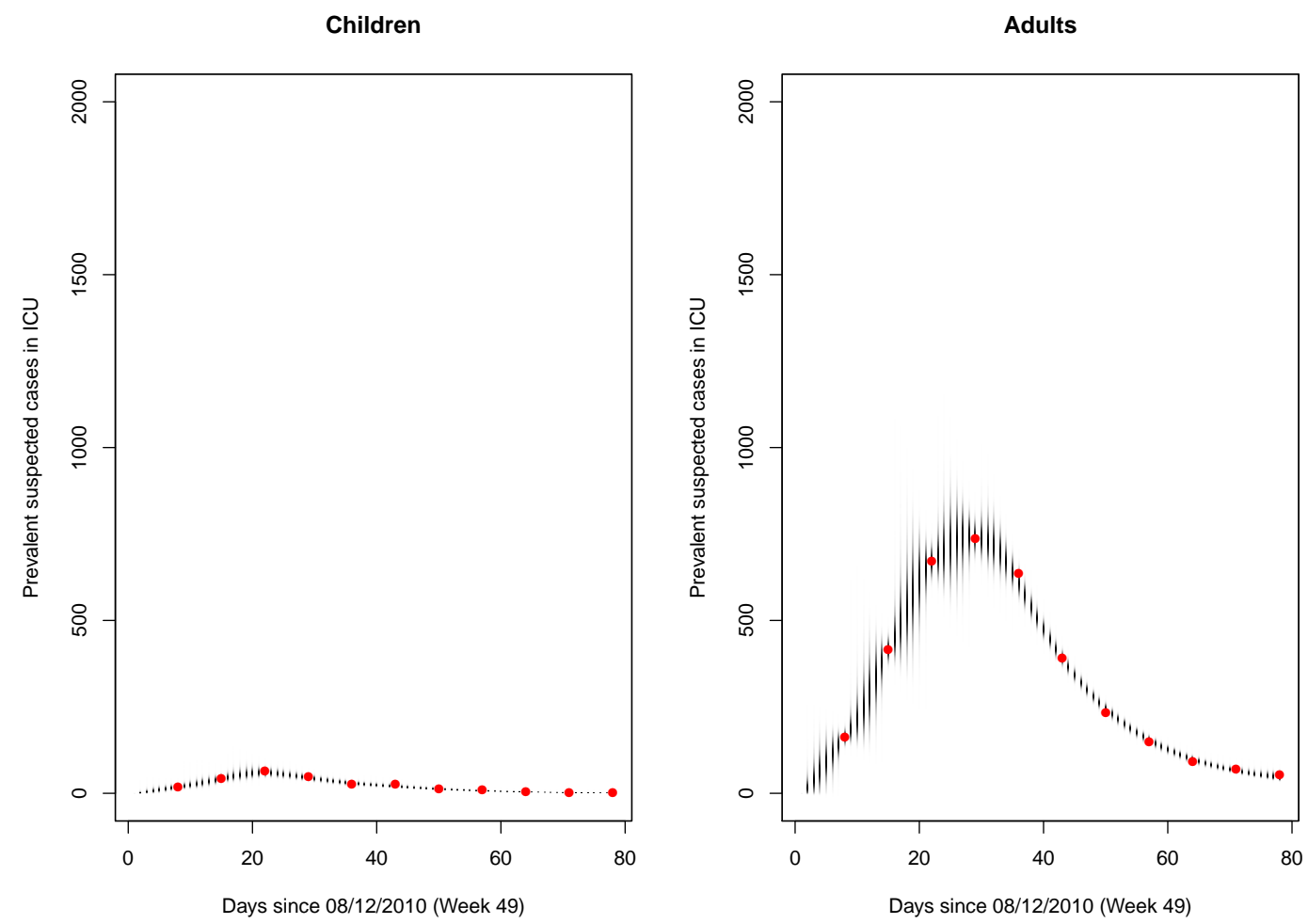

Figure 8: Posterior distribution of prevalent ICU suspected cases (grey) with observed data points (red), by age group.

Reassuringly, the posterior distributions for the expected length of stay $\mathbb{E}\left(L o S_{b}\right)$ have means similar to the prior means given by the analysis of section 4.1, with a posterior mean (sd) of 10.1 (2.1) days for children and 14.0 (2.1) days for adults. These posterior distributions are displayed in Figure 12.

\section{Sensitivity analyses for the first two waves}

Recall that two sets of sensitivity analyses were carried out (Section 6.1 of the main text), to the choice of denominator data and the choice of prior distribution for the infection attack rate. The four analyses with different data informing $I A R_{w, a}$ and $N_{w, a, S}$ are:

1. using the HPA case estimates to inform $N_{w, a, S}$, assuming they do so unbiasedly in both waves (i.e. with $d_{w, a, S}=1$ ), and using no sero-prevalence data;

2. the model presented in the main text and in Presanis et al. (2011), assuming the HPA case estimates are biased downwards and using only the baseline and post-first wave sero-prevalence data;

3. as in model 2, but using all the sero-prevalence data of Table 4 of section 1.1.3, assuming the HPA case estimates are biased downwards in both waves; and

4. as in model 3, but assuming the sero-prevalence data are biased upwards and the HPA case estimates are biased downwards.

Analyses using models 1 and 2 were then repeated using three different prior distributions for the infection attack rate:

a. Dirichlet $(2,2,6)$, allowing the total attack rate over the two waves to be a priori 0.4 on average, with $95 \%$ prior mass in the interval $(0.1-0.7)$, and with a $1: 1$ ratio between the two waves;

b. Dirichlet $(2.67,1.33,6)$, allowing again a prior total attack rate of $0.4(0.1-0.7)$, but with a 2:1 ratio between waves; 


\begin{tabular}{rrrrr}
\hline Week & $\begin{array}{c}\text { Children } \\
\text { Observation }\end{array}$ & $\bar{D}$ Observation & $\bar{D}$ \\
\hline 50 & 19 & 0.95 & 163 & 1.00 \\
51 & 43 & 0.84 & 417 & 0.98 \\
52 & 66 & 1.08 & 672 & 0.99 \\
1 & 47 & 0.54 & 736 & 1.00 \\
2 & 26 & 1.24 & 635 & 1.84 \\
3 & 26 & 1.23 & 392 & 0.34 \\
4 & 13 & 0.43 & 234 & 0.92 \\
5 & 10 & 0.44 & 150 & 0.50 \\
6 & 6 & 0.32 & 93 & 0.79 \\
7 & 2 & 1.03 & 69 & 0.42 \\
8 & 3 & 0.52 & 55 & 1.57 \\
\hline
\end{tabular}

Table 17: Observed in ICU with suspected pandemic A/H1N1 influenza and posterior mean deviance, $\bar{D}$

c. Dirichlet $(1.33,2.67,6)$, allowing a prior total attack rate of $0.4(0.1-0.7)$, with a $1: 2$ ratio between waves.

The sensitivity analyses to the choice of denominator data result in the posterior summaries (median and credible intervals) of the infection attack rate, $I A R$, shown in Figure 13. We find that models 1 and 3 provide the two extremes with respect to the posterior summaries of the IAR. The model presented in the main text, model 2, roughly spans the uncertainty, in all age groups except the over-65s, between models 1 and 3 . Indeed in young children $<5$, the uncertainty from model 2 implies an upper posterior bound greater than that from model 3. Model 4, through making stronger prior assumptions in terms of the sero-prevalence data being biased upwards, results in posterior summaries that are smaller and slightly tighter than model 2 in most age groups, and considerably smaller but of greater uncertainty than model 3. The variation across models in the posterior distribution of $I A R$ results in variation across models in the posterior distribution of the case-fatality risk (Figure 14). However, other than for model 3 (using all the sero-prevalence data and assuming these are unbiased), the upper bounds for the $C F R$ are fairly robust to the choice of denominator data, with most of the variation across models manifesting in the posterior medians and lower bounds.

The sensitivity analyses to the choice of prior distribution for the infection attack rate demonstrate that estimates of both the $I A R$ and the $C F R$ are more robust to this choice than to the choice of denominator data (Figures 15 and 16). Despite the informativeness of the priors for the IAR in sensitivity analyses (a), (b) and (c), there is enough information in the data to dominate any prior knowledge, with the posterior means less than half the prior means and with $95 \%$ credible intervals somewhat tighter than the prior uncertainty. 

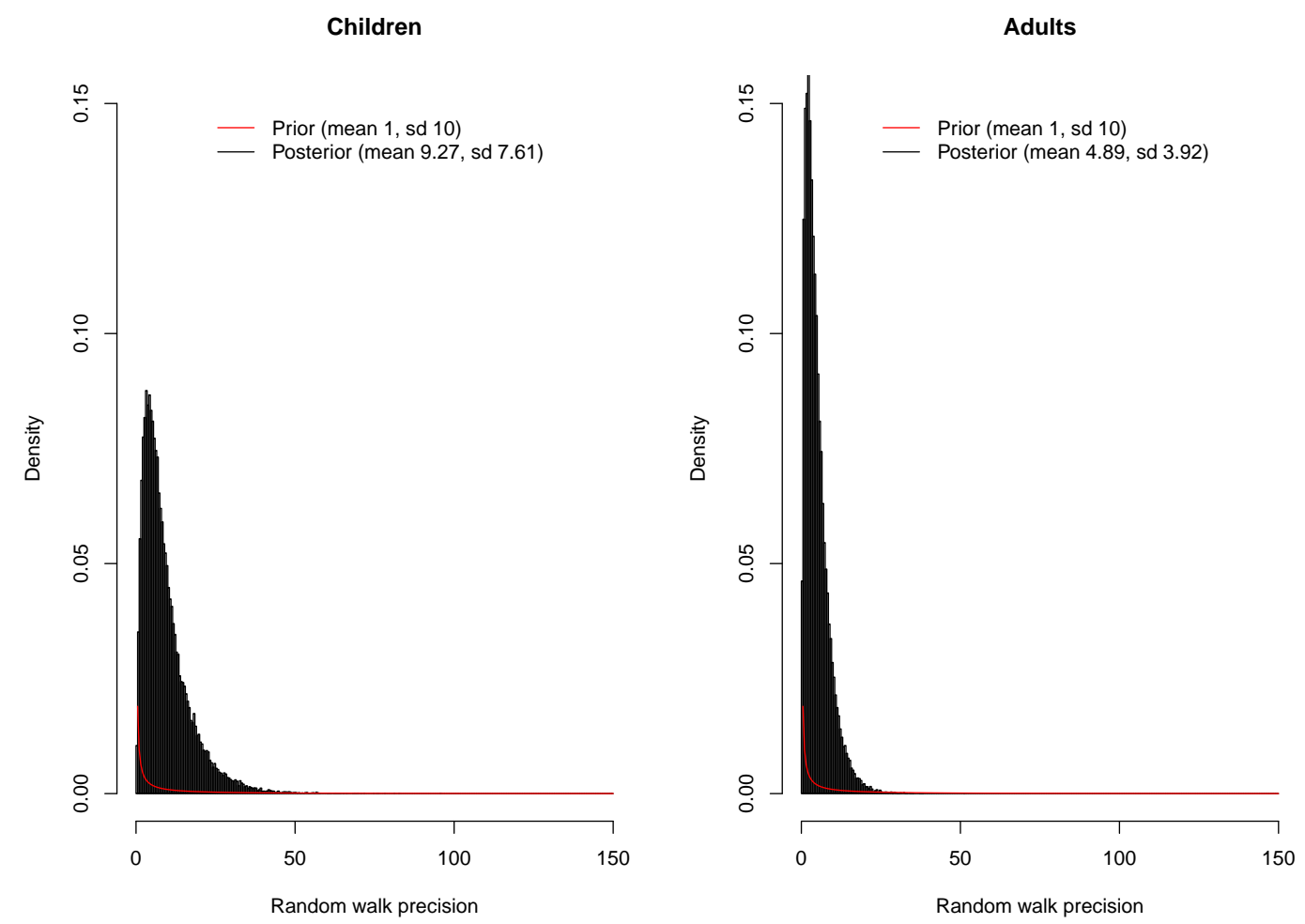

Figure 9: Posterior distribution of random walk precision $\gamma_{R W}$, by age group.
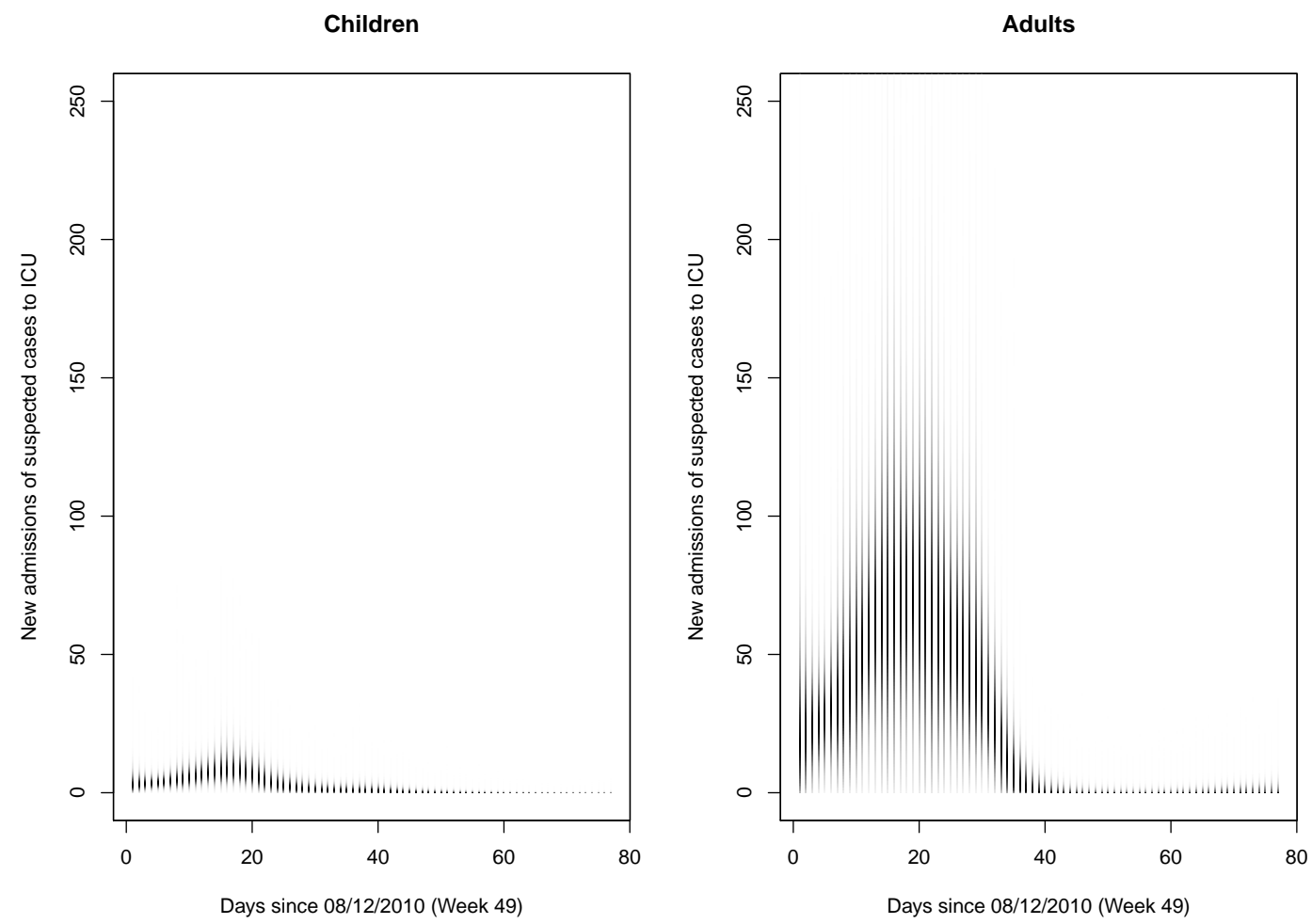

Figure 10: Posterior distribution of $\lambda_{t, b}$, by age group. 

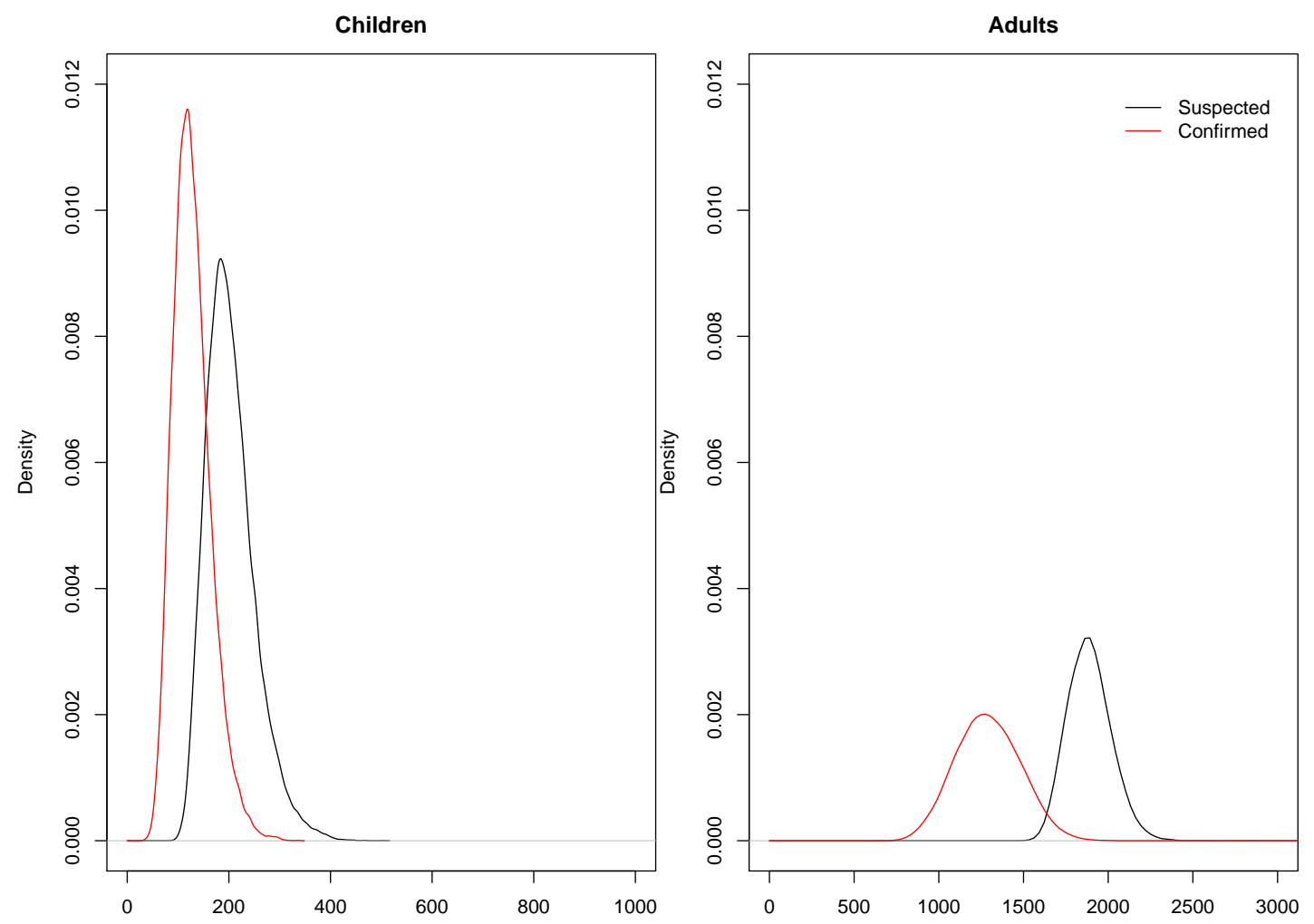

Figure 11: Posterior distribution of $\sum_{t} \lambda_{t, b}$ and of the cumulative sum of the proportion of suspected cases that are confirmed pandemic cases, by age group.
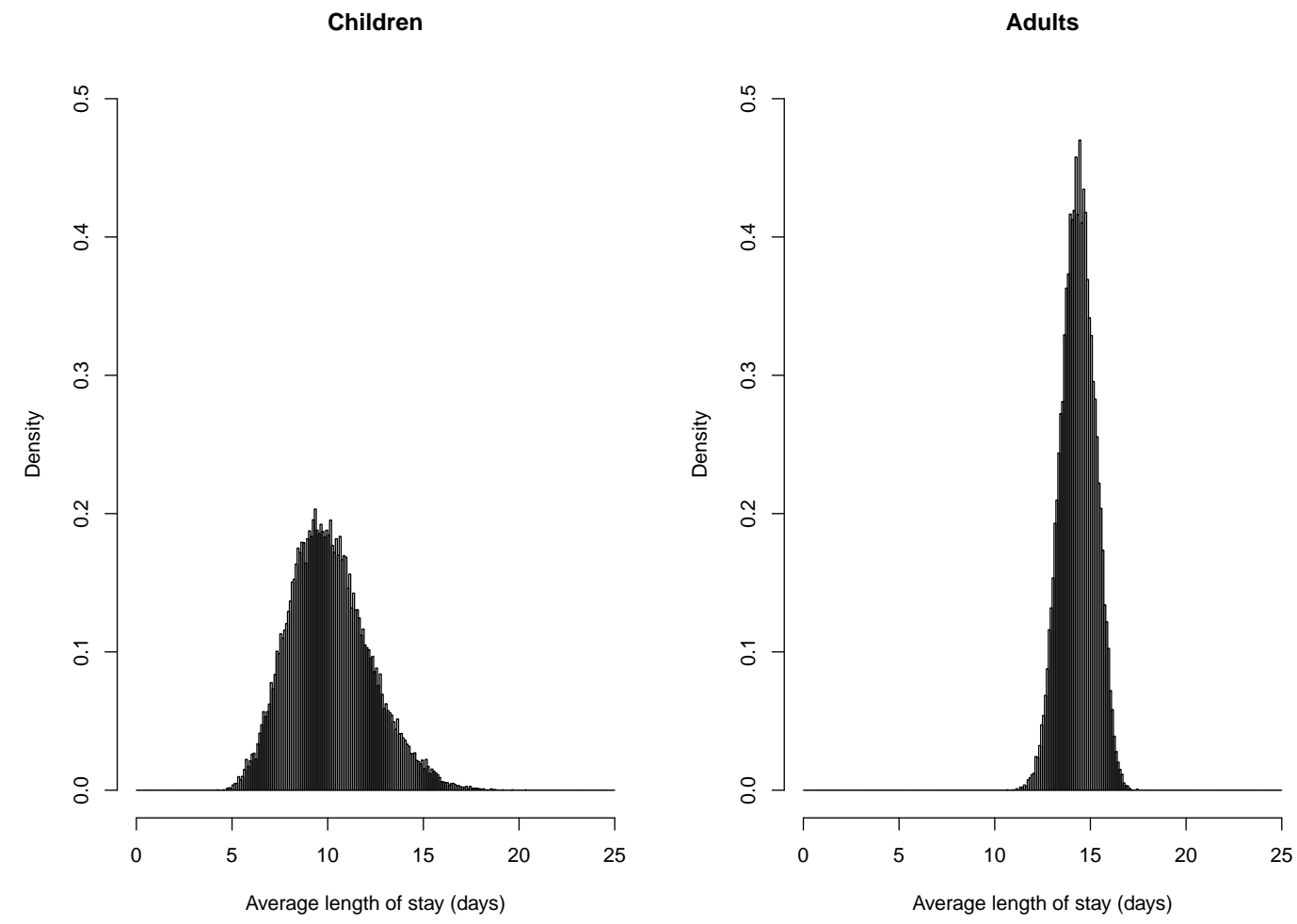

Figure 12: Posterior distribution of $\mathbb{E}\left(L o S_{a}\right)$, by age group. 


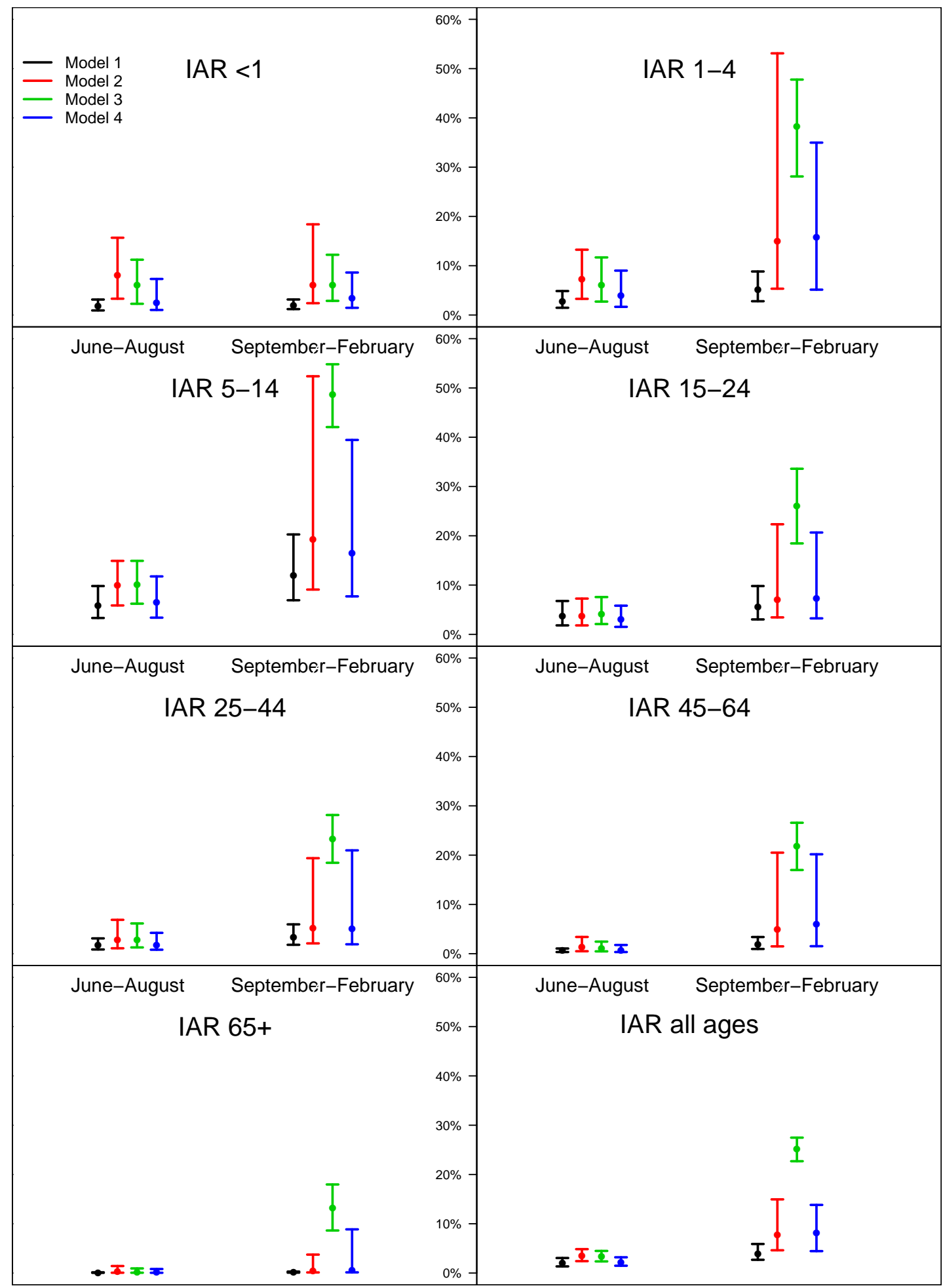

Figure 13: Posterior distribution of $I A R$, from models 1 to 4 , by age. 


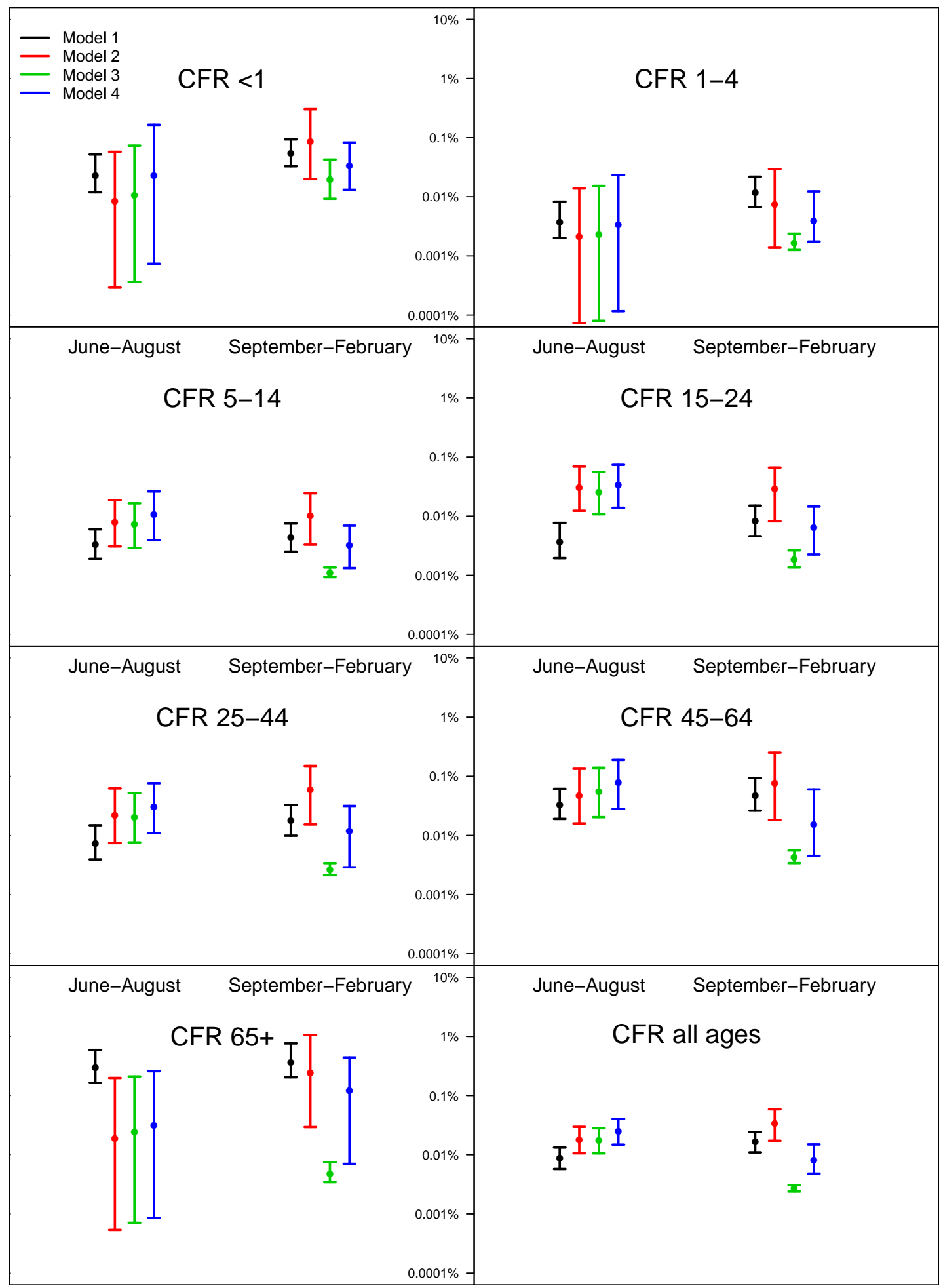

Figure 14: Posterior distribution of $C F R$, from models 1 to 4, by age. 


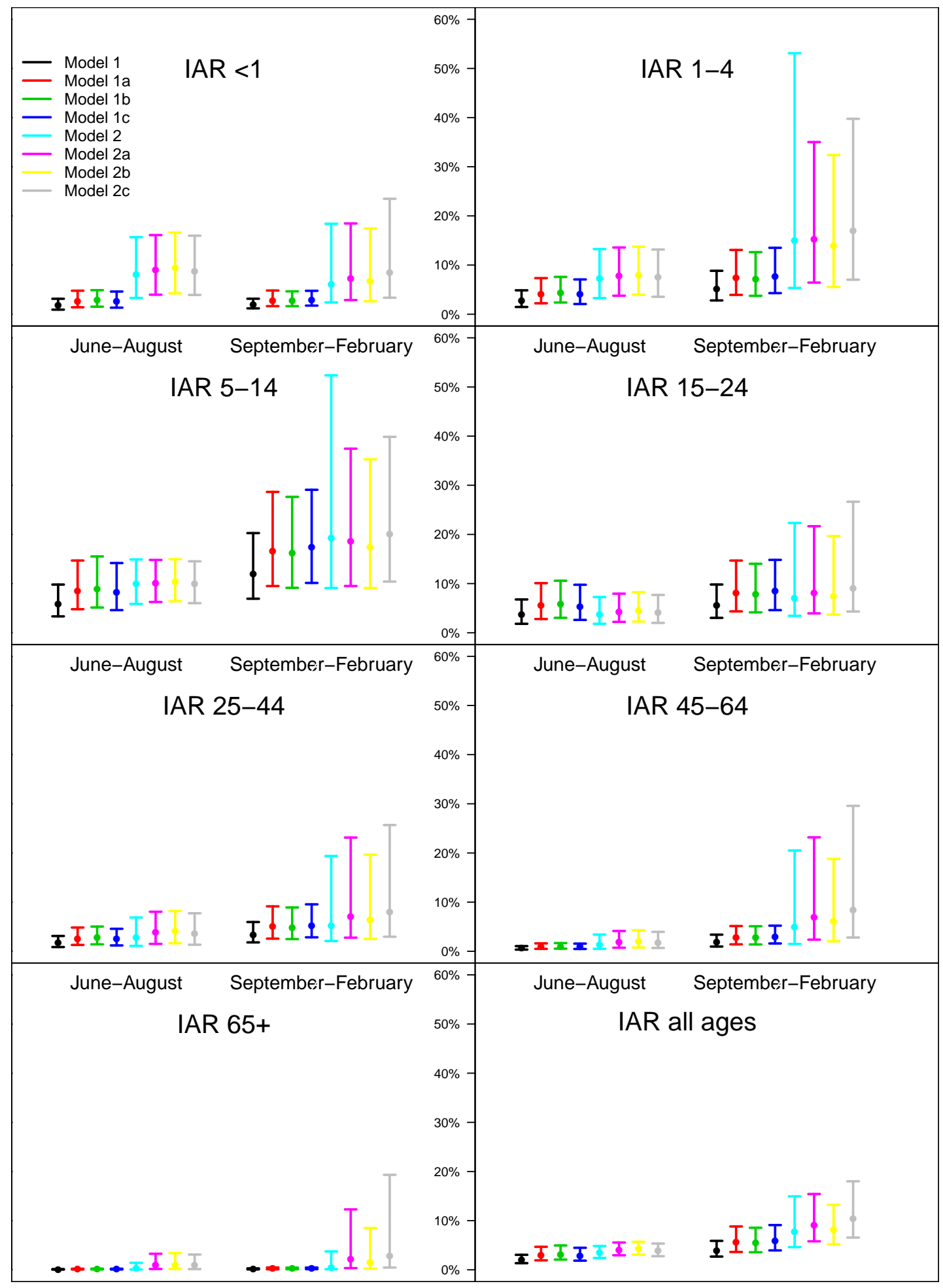

Figure 15: Posterior distribution of $I A R$, from models 1 to $2 \mathrm{c}$, by age. 


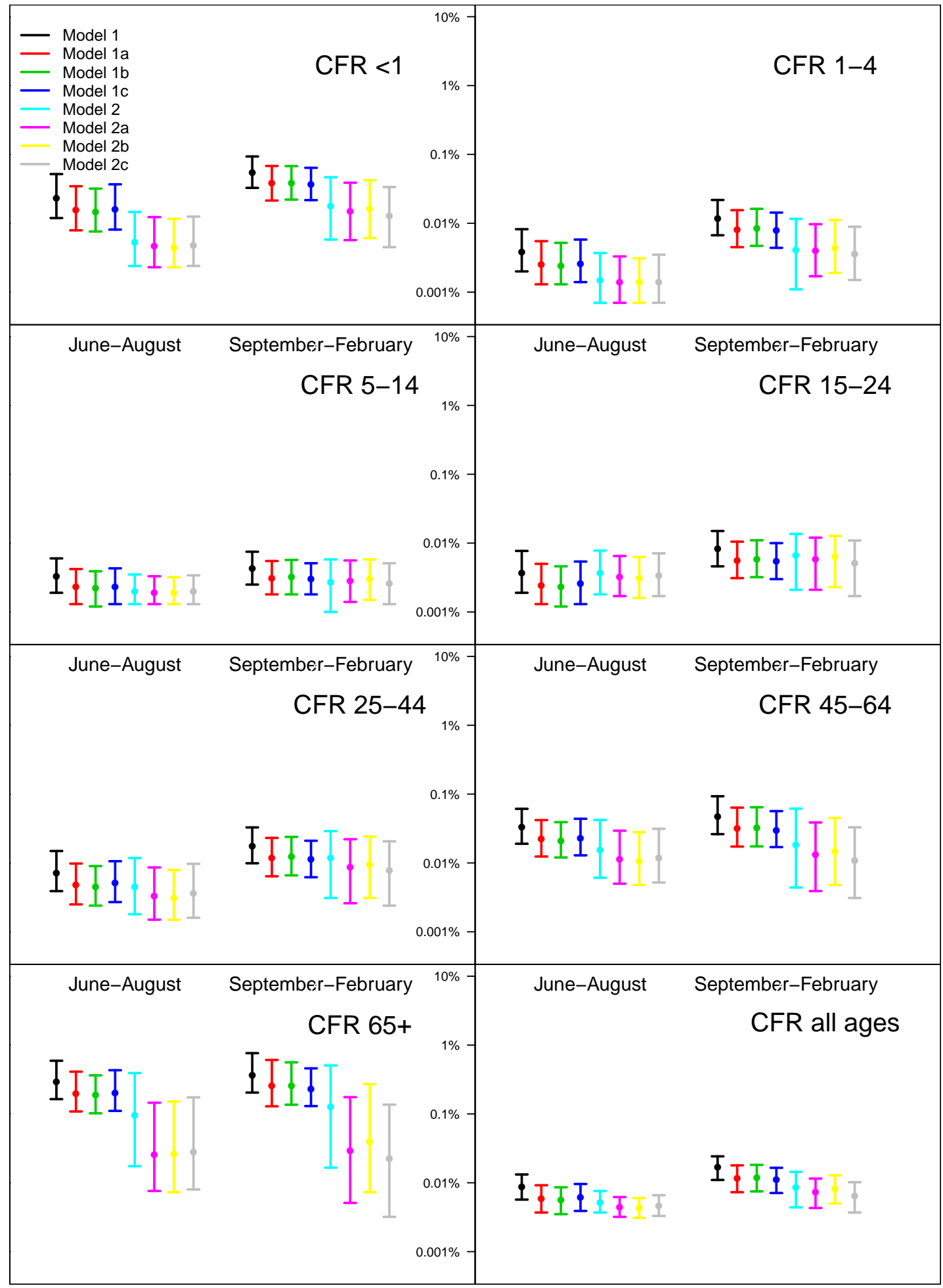

Figure 16: Posterior distribution of $C F R$, from models 1 to 2c, by age. 


\section{References}

Bird, S. M. (2010). Like-with-like comparisons? The Lancet 376(9742), 684+.

Birrell, P. J., G. Ketsetzis, N. J. Gay, B. S. Cooper, A. M. Presanis, R. J. Harris, A. Charlett, X.-S. Zhang, P. J. White, R. G. Pebody, and D. De Angelis (2011). Bayesian modeling to unmask and predict influenza A/H1N1pdm dynamics in london. Proceedings of the National Academy of Sciences 108(45), $18238-18243$.

Brooks, S. P. and A. Gelman (1998). General Methods for Monitoring Convergence of Iterative Simulations. Journal of Computational and Graphical Statistics 7(4), 434-455.

Campbell, C. N. J., O. T. Mytton, E. M. McLean, P. D. Rutter, R. G. Pebody, N. Sachedina, P. J. White, C. Hawkins, B. Evans, P. A. Waight, J. Ellis, A. Bermingham, L. J. Donaldson, and M. Catchpole (2011). Hospitalization in two waves of pandemic influenza A(H1N1) in England. Epidemiology and Infection 139(Special Issue 10), 1560-1569.

Carrat, F., E. Vergu, N. M. Ferguson, M. Lemaitre, S. Cauchemez, S. Leach, and A.-J. J. Valleron (2008). Time lines of infection and disease in human influenza: a review of volunteer challenge studies. American Journal of Epidemiology 167(7), 775-785.

Department of Health (2011). Department of Health Winter Watch. Accessed 25/02/2011.

Donaldson, L. J., P. D. Rutter, B. M. Ellis, F. E. C. Greaves, O. T. Mytton, R. G. Pebody, and I. E. Yardley (2009). Mortality from pandemic A/H1N1 2009 influenza in England: public health surveillance study. BMJ 339(dec10_1), b5213+.

Evans, B., A. Charlett, C. Powers, E. McLean, H. Zhao, A. Bermingham, G. Smith, T. Wreghitt, N. Andrews, R. Pebody, and J. M. Watson (2011). Has estimation of numbers of cases of pandemic influenza H1N1 in england in 2009 provided a useful measure of the occurrence of disease? Influenza and Other Respiratory Viruses 5(6), e504-e512.

Farewell, V. T. (1982). The use of mixture models for the analysis of survival data with Long-Term survivors. Biometrics 38(4), 1041+.

Fleming, D. M. (1999). Weekly Returns Service of the Royal College of General Practitioners. Communicable Disease and Public Health / PHLS 2(2), 96-100.

Grant, A. (2010). Pandemic influenza deaths: capture-recapture estimate. Technical report, Health Protection Agency.

Harcourt, S. E., G. E. Smith, A. J. Elliot, R. Pebody, A. Charlett, S. Ibbotson, M. Regan, and J. H. Cox (2011). Use of a large general practice syndromic surveillance system to monitor the progress of the influenza $\mathrm{A}(\mathrm{H} 1 \mathrm{~N} 1)$ pandemic 2009 in the UK. Epidemiology and Infection FirstView, 1-6.

Hardelid, P., N. J. Andrews, K. Hoschler, E. Stanford, M. Baguelin, P. A. Waight, M. Zambon, and E. Miller (2011). Assessment of baseline age-specific antibody prevalence and incidence of infection to novel influenza A/H1N1 2009. Health Technology Assessment 14(55), 115-192.

Health Protection Agency (2009). Method used to estimate new pandemic (H1N1) 2009 influenza cases in England. Technical report, Health Protection Agency.

Health Protection Agency (2010). Epidemiological report of pandemic (H1N1) 2009 in the UK. Technical report, Health Protection Agency.

Health Protection Agency, Health Protection Scotland, Communicable Disease Surveillance Centre Northern Ireland, and National Public Health Service for Wales (2009). First Few Hundred (FF100) Project: Epidemiological Protocols for Comprehensive Assessment of Early Swine Influenza Cases in the United Kingdom. Technical report, Health Protection Agency.

Hoschler, K., C. Thompson, N. Andrews, M. Galiano, R. Pebody, J. Ellis, E. Stanford, M. Baguelin, E. Miller, and M. Zambon (2012). Seroprevalence of influenza A(H1N1)pdm09 virus antibody, england, 2010 and 2011. Emerging Infectious Diseases 18(11). 
Johnson, S., C. Ihekweazu, P. Hardelid, N. Raphaely, K. Hoschler, A. Bermingham, M. Abid, R. Pebody, G. Bickler, J. Watson, and E. O'Moore (2011). Seroepidemiologic study of pandemic (H1N1) 2009 during outbreak in boarding school, england. Emerging Infectious Diseases 17(9), 1670-1677.

Miller, E., K. Hoschler, P. Hardelid, E. Stanford, N. Andrews, and M. Zambon (2010). Incidence of 2009 pandemic influenza A H1N1 infection in England: a cross-sectional serological study. The Lancet 375(9720), 1100-1108.

Monto, A. S., J. S. Koopman, and I. M. Longini (1985). Tecumseh study of illness. XIII. Influenza infection and disease, 1976-1981. American Journal of Epidemiology 121(6), 811-822.

Osborne, K., N. Gay, L. Hesketh, P. Morgan-Capner, and E. Miller (2000). Ten years of serological surveillance in england and wales: methods, results, implications and action. International Journal of Epidemiology 29(2), 362-368.

Pebody, R. G., E. McLean, H. Zhao, P. Cleary, S. Bracebridge, K. Foster, A. Charlett, P. Hardelid, P. Waight, J. Ellis, A. Bermingham, M. Zambon, B. Evans, R. Salmon, J. McMenamin, B. Smyth, M. Catchpole, and J. Watson (2010). Pandemic Influenza A (H1N1) 2009 and mortality in the United Kingdom: risk factors for death, April 2009 to March 2010. Euro Surveillance 15(20).

Plummer, M. (2010). JAGS Version 2.2.0 User Manual.

Presanis, A. M., R. G. Pebody, B. J. Paterson, B. D. M. Tom, P. J. Birrell, A. Charlett, M. Lipsitch, and D. De Angelis (2011). Changes in severity of 2009 pandemic A/H1N1 influenza in England: a Bayesian evidence synthesis. BMJ 343 . 